\title{
Doing the opposite to what another person is doing
}

\author{
Ivana Bianchi $^{\text {a,* }}$, Ugo Savardi ${ }^{\mathrm{b}}$, Roberto Burro ${ }^{\mathrm{b}}$, Maria Francesca Martelli ${ }^{\mathrm{c}}$ \\ a Department of Humanities, University of Macerata, Via Garibaldi 20, Macerata 62100, Italy \\ b Department of Philosophy, Education and Psychology, University of Verona, Lungadige Porta Vittoria 17, 37129 Verona, Italy \\ c Department of Educational Sciences, University of Macerata, Piazzale Bertelli 1, Macerata 62100, Italy
}

\section{A R T I C L E I N F O}

\section{Article history:}

Received 4 November 2013

Received in revised form 1 June 2014

Accepted 4 June 2014

Available online $\mathrm{xxxx}$

PsycINFO classification:

2323 Visual Perception

2340 Cognitive Processes

2330 Motor Processes

Keywords:

Imitation

Opposition

Gestures recognition

Gestures production

Egocentric space

Allocentric space

\begin{abstract}
A B S T R A C T
The three studies presented here aim to contribute to a better understanding of the role of the coordinate system of a person's body and of the environment in spatial organization underlying the recognition and production of gestures. The paper introduces a new approach by investigating what people consider to be opposite gestures in addition to identical gestures.

It also suggests a new point of view setting the issue in the framework of egocentric versus allocentric spatial encoding as compared to the anatomical versus non-anatomical matching which is usually adopted in the literature.

The results showed that the role of the allocentric system as a key player was much more evident when participants were asked to "do the opposite" as compared to when they imitated which indicates that the two tasks really are different from each other. Response times were also quicker when people "did the opposite" indicating that this is an immediate response and not the result of "reversing an imitation". These findings suggest that the issue of how the oppositional structure of space impacts on human perception and the performance of gestures has probably been underestimated in an area of research which traditionally focuses exclusively on imitation.
\end{abstract}

(c) 2014 Elsevier B.V. All rights reserved.

\section{Introduction}

Being quick to do the opposite of what another person is doing can be crucial when avoiding, for instance, a head-on collision. It is also essential in many situations involving collaborative behavior when two people are face to face, for instance while carrying something, doing surgery or dancing cheek to cheek. And it frequently happens that when two people move toward the same part of the environment one has to move to their left while the other has to move to their right.

Every time two people face each other and "do the same thing", they are at the same time "doing the opposite". When they perform the same action with respect to the environment - i.e. the so-called allocentric frame of reference (for example they both move toward the door/ north) - they are doing the opposite with respect to their bodily coordinates - i.e. the so-called egocentric frame of reference (e.g. one individual moves to their left, the other to their right). Vice versa, when they do the same thing with respect to the egocentric frame of reference they are doing the opposite with respect to the allocentric frame of reference

\footnotetext{
* Corresponding author. Tel.: + 3907332584320

E-mail addresses: ivana.bianchi@unimc.it (I. Bianchi), ugo.savardi@univr.it (U.Savardi), roberto.burro@univr.it (R. Burro), mariafrancesca.martelli@unimc.it (M.F. Martelli).
}

(e.g. when both extend their right hand toward their front-left, for instance to shake hands, they are doing the same but at the same time they are extending their arms toward opposite parts of the environment - i.e. if one is moving north-west, the other is moving south-east).

Over the last 20 years, there has been a vast amount of literature on imitation (e.g.: Bekkering, Wohlschlager, \& Gattis, 2000; Chaminade, Meltzoff, \& Decety, 2005; Genschow et al., 2013; Heyes, 2001, 2011; Heyes \& Ray, 2000, 2004; Meltzoff, 2002; Mengotti, Corradi Dell'Acqua, \& Rumiati, 2012; Prinz, 2002; Watanabe, Higuchi, \& Kikuchi, 2013). In contrast, despite the pervasiveness of oppositional gestures in daily life, there are currently no studies either on whether people perceive that while imitating a gesture they are at the same time doing the opposite or on how this acknowledgment interferes with their perception of doing the same thing (for instance, by slowing down their response time or creating uncertainty). Neither has it been analyzed whether people produce consistent responses when asked to perform opposite gestures or whether this is an indirect task (i.e. people start by figuring out what doing the same would mean and then reverse it) or a direct task which is performed as fast as an imitation task and is not merely a reversal.

This article aims to provide empirical responses to these questions by shifting the focus from studying imitation to studying opposition. In doing this we will suggest a connected theoretical shift: from analyzing 
responses in terms of anatomic versus specular response to analyzing them in terms of allocentric versus egocentric spatial frames of reference. This latter is closer to people's perception of the relations of identity or opposition between body gestures (Bianchi \& Savardi, 2008a, 2008b; Savardi \& Bianchi, 2009).

\subsection{Body and environment: two anchors for oppositional behaviors}

Oppositional behaviors can be anchored to body coordinates or to environmental coordinates. The sagittal, coronal and gravitational body axes (Howard \& Templeton, 1966) are based on three elementary oppositions defined by the structure of the human body: in front of-behind (sagittal axis), left-right (coronal axis) and above-below (gravitational axis). These are basic opposite directions for movements (e.g. walking forward is opposite to walking backward; turning right is opposite to turning left; ascending is opposite to descending) that also define opposite directions for gestures (e.g. pointing in front of you vs. pointing behind you; pointing to the right vs. pointing to the left; pointing up vs. pointing down).

Not only the structure of the human body but also the perceptual structure of the environment is oppositional (Bianchi, Burro, Torquati, \& Savardi, 2013; Bianchi, Savardi, \& Kubovy, 2011; Casasola, 2008; Casasola, Cohen, \& Chiarello, 2003; Savardi \& Bianchi, 2009). Therefore there are at least two different systems which might work as frames of reference to define what "doing the opposite" means: one grounded in human body coordinates (here called the egocentric frame of reference, EGO), the other grounded in the environmental spatial structure (here called the allocentric frame of reference, ALLO).

Many studies have shown that egocentric and allocentric frames of reference are two non-redundant spatial systems that play a role in human perception of space and navigation in space (e.g. Byrne \& Becker, 2008; Holmes \& Sholl, 2005; Klatzky, 1998; Mou, Fan, McNamara, \& Owen, 2008; Mou, McNamara, Rump, \& Xiao, 2006; Mou, McNamara, Valiquiette, \& Rump, 2004; Sholl, 2001; Sorrento \& Henriques, 2008; Waller \& Greenauer, 2007; Wang \& Spelke, 2000). Despite the fact that analyses of imitation have not usually been contextualized within this framework, there is a priori no reason to assume that only one of the two is relevant when describing the spatial relation perceived between two gestures.

In avoidance behaviors between two individuals positioned face to face (e.g. when avoiding a person walking along a street toward you or when sidestepping an opponent in a game of football or basketball), doing the opposite to what the other is doing consists of each person moving quickly away from each other toward two opposite sides of the environment. In these cases it is of secondary importance whether the two people are both moving toward their right (or left) in terms of body-centered coordinates.

Environmental coordinates are also likely to be essential in various other social situations that require quick responses. Think for instance of what happens during a dance or gym class. Instructors implicitly know that by turning their back to their class they make the task of imitation very easy: it is immediately clear which arm or leg the pupils are supposed to move and what direction they are supposed to move in. The only difficulty may be that they can't see the movements made by the instructor well since they are occluded by his/her body. When instructors face their pupils they know that in order to make the imitation quick and easy they have to perform all movements and gestures in an egocentrically opposite way. For example, in order to make them lift their right arms or walk to their right, the instructor has to lift his/her left arm or walk to his/her left. In this case, making a gesture/movement that is quickly perceived by the pupils and the instructor as "the same" (allocentrically the same) implies making egocentrically opposite gestures.

Let us take another example. Imagine that someone walks toward us in the street and asks us directions for a certain road. If we know that this road is right behind us, to the right, we will probably indicate where the road is by stretching out our right arm and pointing behind us. We would then probably see the other person spontaneously looking at and pointing forward in the same direction using their left arm. The person is "doing the same" in terms of environmental coordinates but at the same time is making a contrary gesture in terms of the egocentric body schema. As in the case of the dance class, these are contrary gestures in terms of individual body schemas; there is however also the clear perception that these two people are doing the same thing - and in fact these are all gestures produced with the purpose of imitating another person.

These are only few examples of a long list of everyday actions that stimulate us to ask whether the people involved in these situations perceive that they are "doing the same" or "doing the opposite", both while they perform a gesture themselves and while they observe another person performing the same gesture. When does the allocentric component prevail against the egocentric component and vice versa?

\subsection{What we know from previous studies on imitational and oppositional patterns}

Asking someone to "do the opposite" may seem to be ambiguous, contrasting the (apparent) lack of ambiguity in a request to "do the same". However, as briefly revised here below, previous studies have demonstrated firstly that even imitation is not strictly univocal since it is associated with two different response patterns that not only appear at different developmental phases in childhood but also persist in adult performance (e.g. Avikainen, Wohlschläger, Liuhanen, Hanninen, \& Hari, 2003; Chiavarino, Apperly, \& Humphreys, 2007; Ishikura \& Inomata, 1995; Press, Ray, \& Heyes, 2009). Secondly, when asked to "do the opposite", participants behave consistently and the task is not as ambiguous as one might expect (Bianchi \& Savardi, 2008a).

\subsubsection{Two types of imitation}

Developmental studies (Bekkering et al., 2000; Berges \& Lezine, 1963; Gleissner, Meltzoff, \& Bekkering, 2000; Schofield, 1976) have shown that non-anatomical imitation (also called 'specular imitation' and that is, as we will suggest, a specific case of allocentric imitation) comes more naturally than anatomic (egocentric) imitation until 10 years of age. Anatomic imitation of arm movements represents only $10 \%$ of the total of responses at 8 years, but this increases to $50 \%$ at 13 years and to $80-85 \%$ at 18 years (Wapner \& Cirillo, 1968).

Adults instructed to copy a model's lateralized gestures (see Press et al., 2009) generally used the same side of the body as the model (i.e. anatomical matching). However, only in the case when the model was viewed from behind $\left(0^{\circ}\right)$ was anatomical imitation more accurate than specular imitation. Inversely when adults were rotated $180^{\circ}$ and $240^{\circ}$ with respect to the model, specular imitation was more accurate while no significant differences between the two types of imitation were found at $60^{\circ}, 120^{\circ}$, and $300^{\circ}$. When positioned at $180^{\circ}$ with respect to the model, adults were quicker to non-anatomically rather than anatomically imitate a sequence of ballet poses (Ishikura \& Inomata, 1995). In the same position $\left(180^{\circ}\right)$ participants made more errors when instructed to copy anatomically matching hand and arm actions than when instructed to copy non-anatomically matching limbs (Avikainen et al., 2003; Franz, Ford, \& Werner, 2007). Taken as a whole, these results suggest a) that anatomical matching is not the only imitational response for adults and $b$ ) that at $180^{\circ}$, adults may choose to imitate anatomically even though this is in a sense the most difficult response (Press et al., 2009); they are more accurate and faster when using what in this literature is called specular (or non-anatomical) imitation.

These differences in behavioral performance between anatomical and specular imitation in children and adults have stimulated exploration into whether differences in the activity of the regions of the brain related to imitation are associated with these two types of imitation. Evidence of the critical role of the frontal operculum and posterior parietal cortex in imitation and action observation has been provided by various data (Binkofski et al., 2000; Buccino et al., 2001; Decety, Chaminade, Grezes, \& Meltzoff, 2002; Grafton, Arbib, Fadiga, \& Rizzolatti, 1996; Grezes, 
Costes, \& Decety, 1999; Iacoboni et al., 1999; Koski et al., 2002; Krams, Rushworth, Deiber, Frackowiak, \& Passingham, 1998; Nishitani \& Hari, 2000; Rizzolatti et al., 1996). Researchers have put forward the hypothesis that these two cortical regions may be homologous to those that have been identified as containing mirror neurons in monkeys (di Pellegrino, Fadiga, Fogassi, Gallese, \& Rizzolatti, 1992; Fogassi, Gallese, Fadiga, \& Rizzolatti, 1998; Gallese, Fadiga, Fogassi, \& Rizzolatti, 1996). By investigating cortical activation during both anatomically matching and nonmatching imitations of finger movements, Koski, Iacoboni, Dubeau, Woods, and Mazziotta (2003) found that these two regions were more active during specular imitation than during anatomic imitation. On the basis of this differential activity and of the results emerging from developmental studies, Koski et al. suggested that the major involvement of the mirror neuron system for specular imitation may be interpreted as evidence that the mirror system provides a basic mechanism that allows for some types of simple imitation and that the development of more complex imitative skills (i.e. anatomical imitation) may emerge as a function of the maturation of other systems which are important for visuospatial transformations.

\subsubsection{Opposition}

In the literature mentioned thus far, participants have always been asked to imitate, either using the same anatomical arm or "reflecting" like a mirror, i.e. using the non-anatomically matching arm. Participants have never been asked "to do the opposite". This last request is potentially open to various solutions. The implication is not merely that one must use "the opposite arm", in fact in this latter case it would anyway be necessary to specify "opposite with respect to what" i.e. body coordinates or environmental coordinates. The opposite of a simple gesture like "right arm, still, tensed, held out straight in front of the body" might be in theory "right arm, still, bent, tensed, held out in front of the body" as well as "right arm, still, tensed, held out straight behind the body" or "left arm, still, tensed, held out straight in front of the body", or even "left arm, moving back and forth, relaxed, held out straight behind the body" and so on. In other words, if imitation apparently implies merely a replication of all the properties of the target gesture, "doing the opposite" requires an apparently arbitrary decision about which property (or properties) to make opposite.

Pilot studies with 6 to 8 year old children and undergraduate students who were asked to "do the opposite" of a simple arm gesture showed that the task is easily understood and consistently resolved by both age groups following some "rules" (Bianchi \& Savardi, 2008a, pp. $101 \mathrm{ff}$.). The vast majority of participants (around 90\%) made only one or two characteristics of the initial gesture opposite, usually its direction (i.e. changing a forward movement into a backward movement) or its lateralization (i.e. changing the arm used); less than $5 \%$ of participants turned a leg gesture into an arm gesture or transformed aspects concerning the "shape" of the gesture (e.g. bent as opposed to straight or vice versa) and less than $5 \%$ used an additive strategy transforming more than one of the characteristics of the gesture (for example, lateralization together with direction, together with bent versus straight arm or closed fist/open hand). ${ }^{1}$

Moreover, these pilot studies suggested that the importance of the egocentric or allocentric frames of reference differed in coronal and

\footnotetext{
1 This is consistent with what was found when the task was applied to visual non-motor configurations: when asked to draw the opposite of a simple two dimensional figure, both adults and 7-8 year old children modified only one or two characteristics of the initial figure (Bianchi \& Savardi, 2008b). For example, participants inverted the figure from upright to upside down rather than change the shape (e.g. from round to angular), color (e.g. from black to white) or its size (e.g. from big to small). This non-additive strategy was also found in recognition tasks: configurations which differed in terms of more than two properties were perceived by participants as different and not opposite; the single transformation associated with the recognition of opposition was usually a reversal of the figure's direction (with figures clearly showing a direction) or a transformation of the main axis of orientation from horizontal to vertical (or the other way round) when the figure did not point in a particular direction.
}

sagittal gestures (Bianchi \& Savardi, 2008a). This also emerged from studies investigating the relationship perceived between one's own gesture and the reflection of a gesture in a plane mirror (Bianchi \& Savardi, 2008c, 2009).

\section{From anatomic versus specular matching to egocentric versus allocentric frames of reference}

The two types of imitation described in the previous section are usually called "anatomical matching" (i.e. a movement made with the right arm is imitated using the right arm) and "non-anatomical matching" (i.e. a movement made with the right arm is imitated with the left arm). The latter is also referred to as "specular imitation" in reference to the left-right reversal shown in reflections in plane mirrors. Describing non-anatomical matching in terms of specular imitation is unambiguous for certain positions of an observer with respect to the model, namely at $180^{\circ}$ when the situation corresponds to when a mirror is frontoparallel to an observer. But when a model and an observer are oriented in other ways, a direct matching with the real patterns created by plane mirrors is not available. In these cases "specular" means "nonanatomically matched" in the sense of using the right side when the model is using the left side and vice versa. Here the term "specular" is not useful from an operational point of view and is even a bit misleading. It is for this reason that we suggest avoiding this term. However, neither is the distinction between anatomical and non anatomical gestures sufficient since, although familiar and apparently theoretically parsimonious, it hides important differences between the spatial identity of gestures which are equally either matching or non-matching from an anatomical point of view, as we will see below. To avoid this limit we propose describing the spatial identity of gestures in terms of egocentric versus allocentric frames of reference. There are at least four reasons, as listed below, which justify this shift.

1) As already mentioned in Section 1.1, there is widespread evidence that these two frames of reference are essential in the perception and memory of spatial stimuli and gestures are in fact spatial stimuli. In Fig. 1 it is immediately evident that both the egocentric and allocentric frames of reference play a role in the spatial relationship perceived between the gestures: everyone is doing the same in an anatomic/egocentric sense (i.e. everyone has their right arm extended forward) and in this sense would all equally be defined as anatomical imitations. However, whereas the gesture of the woman in

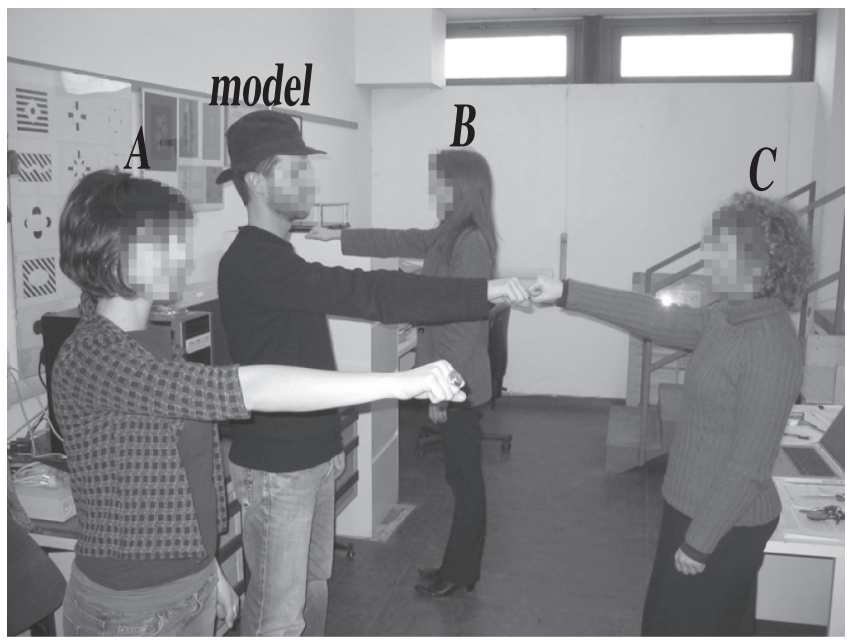

Fig. 1. The people are all making the same gesture in terms of egocentric coordinates (they have their right arm extended forward). However while the gestures that person A and the man with the hat (the model) are making seem to be the same, those made by $B$ and $\mathrm{C}$ seem to be opposite to the model. With respect to the environmental coordinates, $\mathrm{B}$ and $\mathrm{C}$ are in fact pointing in the opposite direction (whereas $\mathrm{A}$ and the model point in the same environmental direction). 
condition A appears to be absolutely identical to that of the model, in conditions $B$ and $C$ the gesture appears to be contrary since it points in the opposite direction. Is it appropriate to ignore all this information or might it rather be that this different perceptual structure is crucial to the participant's response?

When given a non-specific instruction such as "do the opposite" (or "do the same") with respect to what a model is doing, people will produce different responses depending on whether they recognize the target gesture as "the model is extending his/her right arm forward" (an egocentric encoding of the gesture) or "the model is extending his/her arm on this side of the room and is pointing toward the other side of the room" (an allocentric encoding). While the idea of egocentric encoding of a gesture corresponds to that of anatomical matching (i.e. the gesture is identified based on the person's body schema), the idea of allocentric encoding does not overlap with the definition of non-anatomical matching. As shown in Fig. 1, the identification of the gesture in terms of the allocentric frame of reference holds for anatomically matching gestures as well.

2) It has been proved that egocentric and allocentric frames of reference are effective in terms of explaining the description that adults give regarding the relationship perceived between their body and their reflection (Bianchi \& Savardi, 2008c); the relationship between a body and its reflection in a mirror is simply a subset of all the possible spatial positions and orientations between two human bodies.

3) The two patterns of imitation found in the literature and discussed in Section 1.2.1 (usually referred to in terms of anatomical versus specular matching) can be easily re-described and explained in terms of allocentric versus egocentric frames of reference. This also covers conditions which are not suitably described in terms of specular matching, as emphasized above. Look at Fig. 2. When participants in an experiment view a model from behind (Fig. 2a, b), this corresponds to the $0^{\circ}$ rotation condition in the studies described in Section 1.2.1. In Fig. 2a, both $t$ and $p$ move their right arm which means that they are doing the same thing anatomically (egocentric frame of reference) but they are also "doing the same" with respect to the environment, i.e. both people move their arm toward $\mathrm{X}$ (allocentric frame of reference). The two frames of reference are thus consistent. In this case, as found by Press et al. (2009), unsurprisingly participants turned out to be quick and accurate when asked to "do the same" anatomically (which is also consistent with "doing the same" allocentrically). This was not so when they performed non-anatomically matching gestures, i.e. when the participants were asked to extend their left arm toward the left while the model extended his/her right arm toward the right (Fig. 2b). This is not surprising either: what participants were asked to do was not an imitation from the egocentric point of view, but neither was it an imitation from the allocentric point of view - in fact the participant was asked to extend his/her arm toward the opposite side of the environment $(\mathrm{Y})$ with respect to where the model was extending their arm (X). It is hard to think of this gesture in terms of imitation and simply defining it as non-anatomical does not convey the idea that this gesture is precisely the opposite from an allocentric point of view (notice that this is not necessarily true for all nonanatomical gestures - e.g. Fig. 2d).

When the model faces the participant (this condition was referred to as $180^{\circ}$ rotation in Avikainen et al., 2003; Franz et al., 2007; Ishikura and Inomata, 1995; Press et al., 2009), the two frames of reference are no longer congruent. As shown in Fig. 2c, doing the same anatomically (i.e. egocentric imitation: both $p$ and $t$ stretch out their left arm to the side) means producing a gesture which is opposite in terms of allocentric space: the target moves toward Y, the participant toward X. Conversely, producing a non-anatomical imitation (Fig. 2d) means making the same gesture allocentrically (i.e. both people extend their arms toward Y) but the opposite gesture egocentrically (i.e. $t$ moves his/her left arm on his/her left and $p$ moves his/her right arm on his/her right). In the literature cited above, the response represented in Fig. $2 d$ turned out to be easier. If we do not take into account the allocentric frame of reference and we limit ourselves to the anatomical versus non-anatomical definition, we cannot see what Fig. 2a (anatomical gesture) and Fig. 2d (nonanatomical gesture) have in common which is that in both cases $p$ is imitating $t$ with respect to the external environment (allocentric imitation).

4) A final reason to adopt allocentric versus egocentric frames of reference in descriptions rather than anatomical versus non-anatomical classifications is that in the three studies presented here the task was to "do the opposite" not to imitate a gesture using the anatomically non-matching arm (as was instead the case in Avikainen et al., 2003; Franz et al., 2007; Press et al., 2009) with in effect only two possible responses as represented in Fig. 3a, and b. We need to foresee at least four possible alternative responses to the same target gesture (Fig. 3c, d, e, f), since which arm a participant uses might co-vary with a change of direction. This means going beyond the limits of a specular pattern - as well as of anatomical vs. nonanatomical classifications.

\section{Study 1}

The first study was designed to establish whether "doing the opposite" can be considered simply the reverse of "doing the same" or whether differences would emerge between the two tasks indicating that the allocentric and egocentric frames of reference play a different role in the two tasks. Two groups of participants took part in the study. One group was asked to "do the opposite" of the gestures made by a model. As a control, a second group of participants were asked to "do the same" as the model. We decided to study the task variable

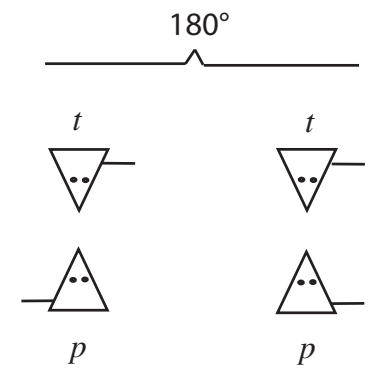

C

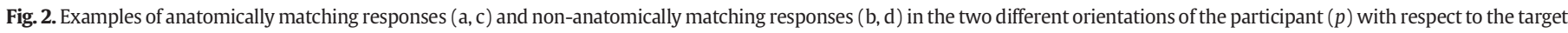

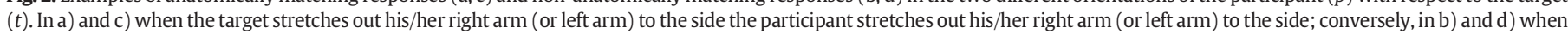
the target stretches out his/her right arm to the side the participant stretches out his/her left arm or vice versa. 

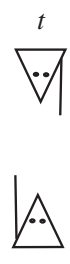

$p$
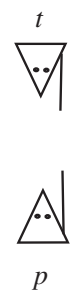
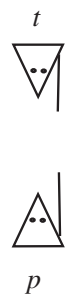
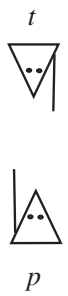
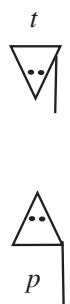
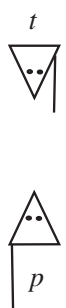

ANATOMICAL MATCHING NON-ANATOMICAL MATCHING

$t=$ left arm in front

$p=$ left arm in front $t=$ left arm in front

$p=$ right arm in front
LATERALIZATION x DIRECTION:

EGO: $t=$ left arm in front

$p=$ right arm in front

ALLO: $\mathrm{t}=$ east arm towards south $p=$ east arm towards north
LATERALIZATION x DIRECTION:

$E G O: t=$ left arm in front

$p=$ left arm in front

ALLO: $\mathrm{t}=$ east arm towards south $p=$ west arm towards north

d
LATERALIZATION x DIRECTION:

$E G O: t=$ left arm in front

$p=$ right arm behind

ALLO: $\mathrm{t}=$ east arm towards south $p=$ east arm towards south

e
LATERALIZATION x DIRECTION:

EGO: $t=$ left arm in front $p=$ left arm behind

ALLO: $\mathrm{t}=$ east arm towards south $p=$ west arm towards south

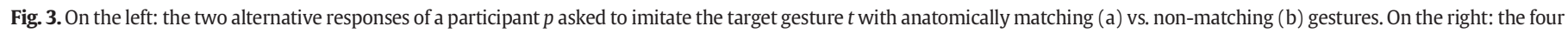

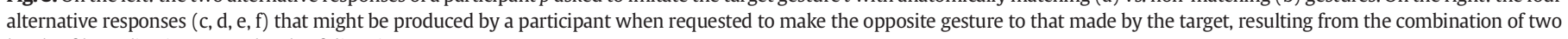
levels of lateralization $\times$ two levels of direction.

between subjects to avoid any tendency in participants to respond to one task with a mechanical inversion of the response for the other task. If "doing the opposite" is simply the reversal of "doing the same", no significant differences should emerge between one frame of reference or the other in the two tasks.

A comparison between average confidence ratings associated with responses in the two tasks provided information on any uncertainty associated with "doing the opposite" as compared to "doing the same".

In addition to the type of task variable (studied between subjects), three further independent variables were studied within subjects: gesture (coronal, sagittal, and oscillation), orientation (homo-directional and counter-directional), and localization (in front, to the side and behind the model). If participants respond by merely applying a rationally chosen criterion (e.g. egocentric imitation/opposition), no difference should emerge when the localization or orientation of the participant with respect to the model is changed, or when the type of gesture is altered. If, conversely, a change occurs, this suggests that the specific spatial configuration counts.

With regard to the gesture variable, we considered both bilateral gestures made with both arms (in order to control the variable lateralization of the gesture and to study only the effect of direction) and a gesture made with one arm (to enable a study of the role of lateralization).
CORONAL GESTURE
SAGITTAL GESTURE

OSCILLATION

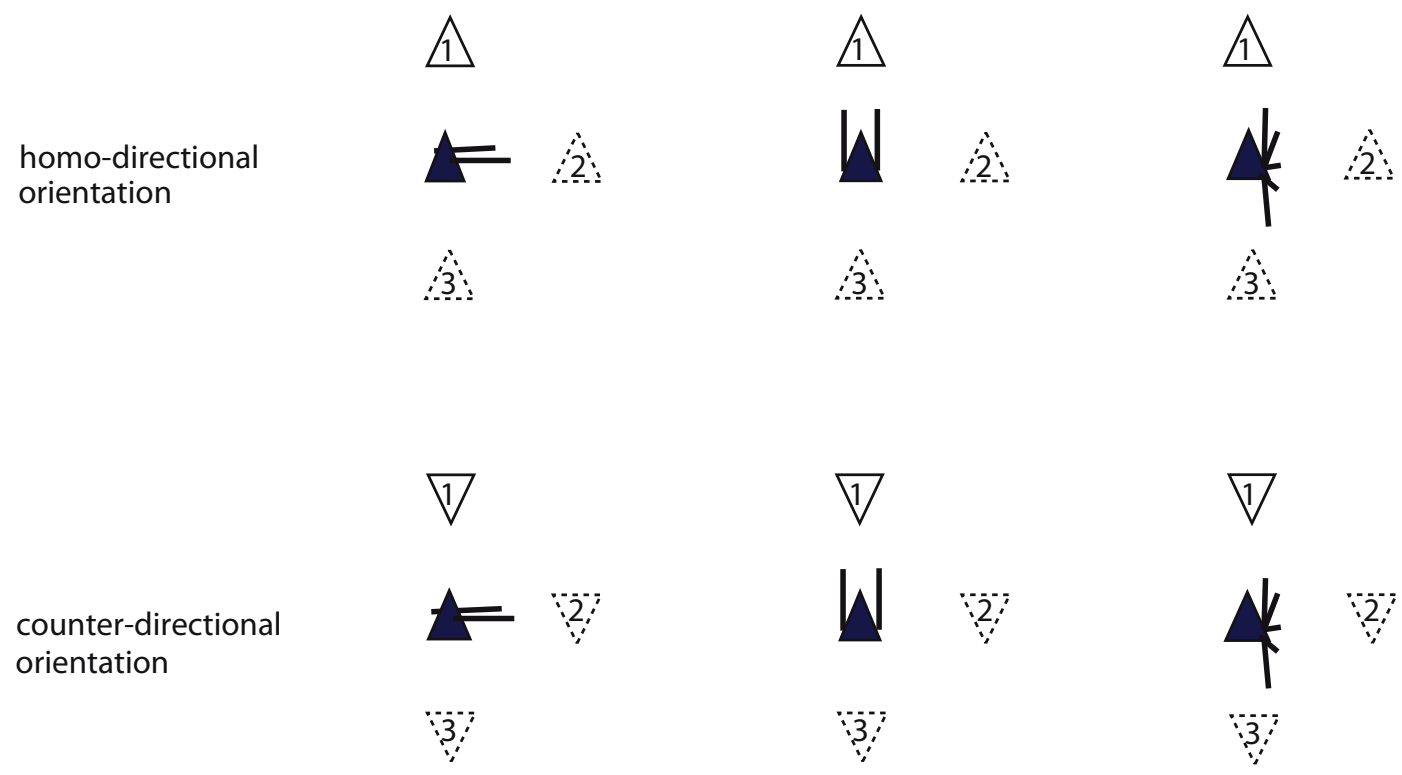

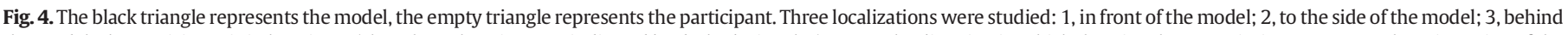

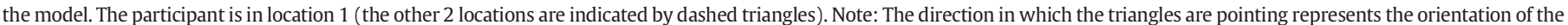

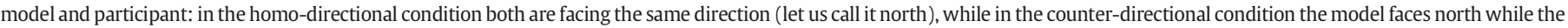

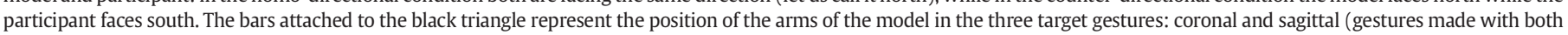
arms) and oscillation (gesture made with the right arm). 
The bilateral gestures that we considered were coronal and sagittal (the three different gestures are represented, in columns, in Fig. 4). Given the shape and biomechanics of the human body, front and back (sagittal opposites) are more asymmetrical than left and right (coronal opposites). Therefore "arms extended to the left" and "arms extended to the right" are gestures which are visually more similar than "arms extended forward" and "arms extended behind". For this reason we expected the egocentric frame of reference to be more relevant for sagittal than coronal gestures. For gestures made with one arm we used oscillations (namely, two oscillations back and forth, covering an angle of approximately $30^{\circ}$ ). These were made with the right arm. Oscillation is a gesture with a weak orientation (it involves more than one direction) and therefore the arm which is used (lateralization) should be the aspect which emerges perceptually.

With regard to the localization variable, participants were positioned in front of, to the side, and behind the target (the three locations are represented, in Fig. 4, by the numbers 1, 2 and 3). They were oriented either homo-directionally with regard to the model (e.g. they both faced north, see first row in Fig. 4) or counterdirectionally (e.g. the model faced north, the participant faced south, see second row in Fig. 4). In the counter-directional orientation the two frames of reference are inconsistent and thus each response satisfies either one or the other. In the homo-directional orientation, the egocentric and allocentric frames of reference work consistently and it is not possible to distinguish which frame of reference participants are using in their responses. However, we decided to study this condition too because we were interested in comparing the confidence ratings in the homo-directional and counter-directional conditions in order to establish a further index of the importance of both frames of reference. In fact, if both count, the confidence of the participants should be greater when the two frames are consistent (i.e. following one frame of reference means at the same time also satisfying the other) than when the two frames conflict (i.e. responding according to one frame of reference necessarily means contrasting the other).

\subsection{Method}

\subsubsection{Participants}

73 undergraduate students divided into two groups participated in the study: 36 (mean age 21.4) participated in the "do the opposite" task (group 1); 37 (mean age 21.1) in the "do the same" task (group 2).

\subsubsection{Procedure}

The experiment was conducted in an empty room at the University of Macerata. Participants took part in the experiment individually. An assistant to the researcher played the role of the model and performed the three gestures (coronal, sagittal, and oscillation), one at a time. The model occupied the same location at the center of the room for the entire duration of the study, whereas the participant changed his/ her location and orientation (as represented by the white triangles in Fig. 4).

Participants were asked to observe the gesture made by the model and "do the opposite" (group 1) or "do the same" (group 2). It was made clear that there were no right or wrong responses and that they had to give an immediate and spontaneous response. After they had produced a gesture, they were then asked to rate their confidence in the response given using a 5-point scale (ranging from $0=$ not at all confident to $4=$ totally confident). Responses were recorded by the experimenter on a response sheet where simple diagrams representing the gestures made by the target were drawn and where the experimenter drew the movement made by the participant and reported the confidence rating expressed. The experimental sessions were also video-recorded.

\subsubsection{Stimuli}

Eighteen stimuli were presented to each participant ( 3 gestures $\times 3$ locations $\times 2$ orientations) in random order. The same random order was used for both groups. There was one single trial for each gesture; participants thus responded in total to 18 different conditions.

\subsection{Results}

Diagrams representing the allocentric (ALLO) and egocentric (EGO) responses for every gesture in each single location were prepared by the experimenters in order to facilitate the coding of responses. Responses that did not fit in with either of the two types were classified as "other". These were for instance cases where participants responded to a coronal gesture by raising their arms, or to a sagittal gesture with a coronal movement, e.g. by stretching out both their arms to the sides. These "other" responses were not expected to be frequent and for this reason they were not coded into further different categories. All responses fitted in with the three categories (EGO, ALLO, other) thus no data were removed. Two independent judges coded participants' responses using both the response sheets and the videos. The inter-rater agreement turned out to be excellent (Cohen's $\kappa=0.93$ ).

\subsubsection{Overall indications regarding the frame of reference used}

As mentioned in the Introduction section, only the counterdirectional orientation allows us to distinguish between responses since in this condition the responses which are consistent with the egocentric frame (EGO) are in contrast with the allocentric frame (ALLO) and vice versa. Thus, only the responses for the counter-directional condition are useful in the following analysis.

The overall frequency of allocentric versus egocentric responses (independently of the specific localization and gesture) significantly differed in the two tasks $\left(\chi^{2}=91.748\right.$, df $\left.=1, \mathrm{p}<0.001\right)$. Around $80 \%$ of responses in the imitational task were egocentric and only $20 \%$ were based on the allocentric space (Fig. 5, top-left diagram). This is in line with the preference for egocentric responses found in the literature on imitation tasks for adults (Press et al., 2009, Study 1). In contrast, when asked to "do the opposite" (Fig. 5, top-right diagram), the egocentrically based responses dropped to $41 \%$ while allocentrically based responses were significantly more frequent (54\%).

We tested whether the different frequency of egocentric and allocentric responses in the two tasks was confirmed within participants. $^{2}$ To do this, we eliminated the "other" response for each participant and then calculated the proportion of EGO and ALLO responses in relation to the total number of responses remaining. A mixed effect model ANOVA was then conducted to compare the average ratio of EGO and ALLO responses in the two tasks. As shown in Fig. 5 (bottom graph), a significant interaction between the two variables was found $\left(F_{(1,71)}=42.998, \mathrm{p}<0.001\right)$ : the proportion of allocentric responses was significantly higher when participants were asked to "do the opposite" as compared to when they were asked to "do the same". In contrast, the proportion of egocentric responses was significantly higher when participants were asked to "do the same" as compared to "doing the opposite".

\subsubsection{Effects of the variables (task, type of gesture, and localization) on the} frequency of egocentric and allocentric responses

A mixed effect regression model was conducted to study whether the frequency of EGO and ALLO responses varied based on the type of gesture, the localization considered and the task (with EGO/ALLO as a dichotomous dependent variable; task, type of gesture and localization

\footnotetext{
2 We generally analyzed data (overall frequency data, average confidence ratings, average response times) for the conditions studied and "collapsed" them across participants, since the conditions were the object of the study. However, when we felt it was meaningful, we sometimes also analyzed responses for each participant and collapsed them across the conditions.
} 

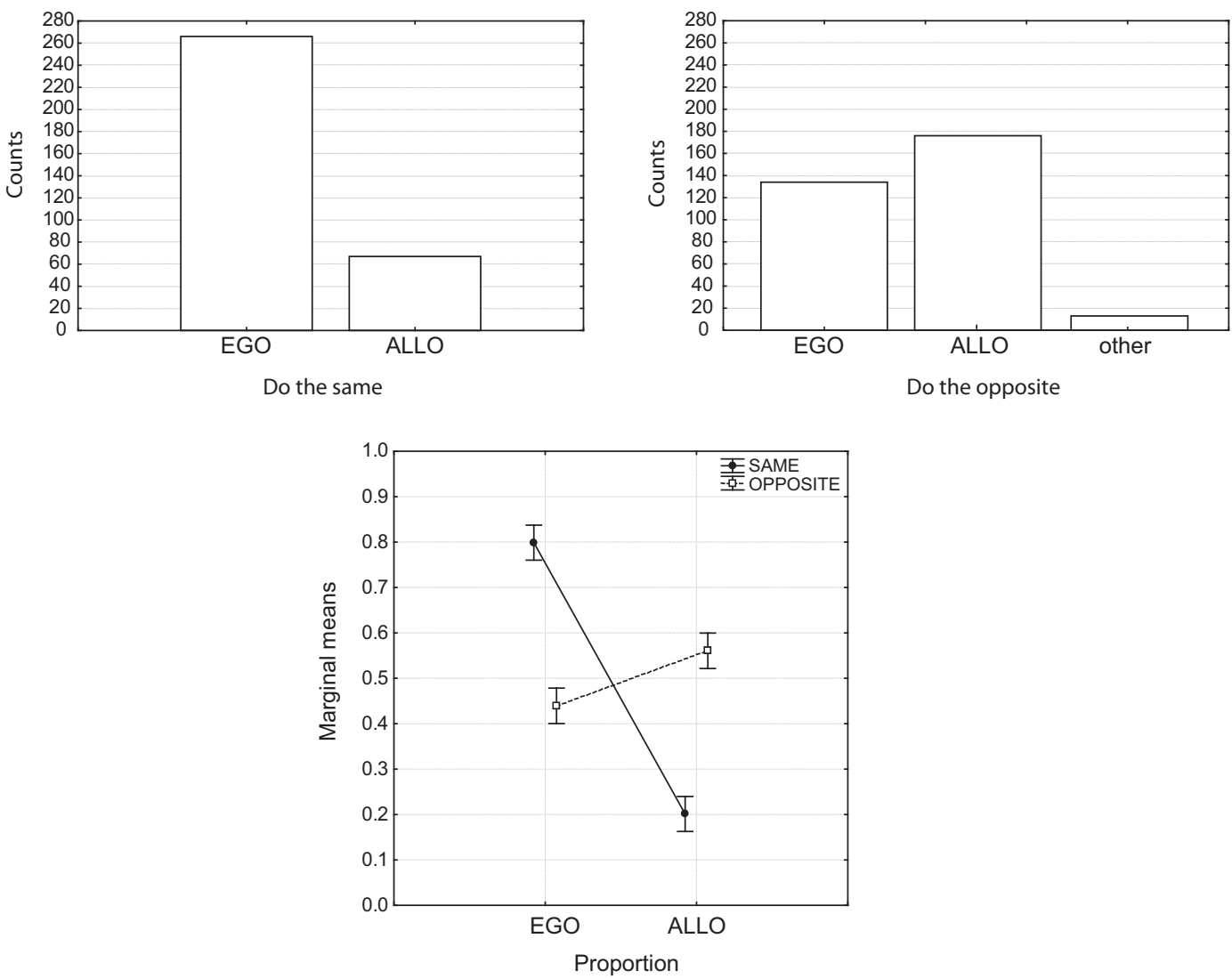

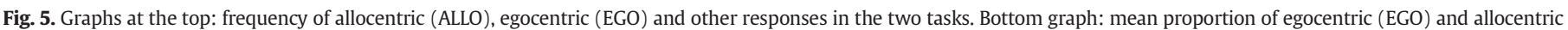
(ALLO) responses for participants taking part in the "do the same" and "do the opposite" tasks. All graphs refer to responses collected in the counter-directional condition.

as fixed effects and participants as a random effect nested in task). For the reasons explained previously only data from the counterdirectional condition were considered in this analysis.

The analysis revealed: a ) a significant effect of task $\left(\mathrm{F}_{(1,71)}=44.530\right.$, $\mathrm{p}<0.001$ ) confirming that egocentric responses were significantly more frequent when participants were asked to "do the same" as compared to "doing the opposite" (and vice versa for allocentric responses); b) a significant effect of gesture $\left(F_{(2,139)}=20.621\right.$, $\left.p<0.001\right)$ : the frequency of egocentric responses was particularly high for sagittal gestures, less frequent for oscillation and even less for coronal gestures.
However, as indicated by the interaction between gesture and localization $\left(\mathrm{F}_{(4,273)}=5.965, \mathrm{p}<0.001\right)$ and between task, gesture and localization $\left(F_{(4,273)}=2.232, \mathrm{p}=0.05\right)$, these conclusions need to be refined. In fact, as Fig. 6 shows and the Bonferroni post-hoc tests confirmed ( $p<0.02$ ), participants gave a particularly high number of egocentric responses when they were asked to make "the same" sagittal gestures in all three localizations and to make "the same" oscillation or coronal gesture when standing to the side of the model (i.e. in localization 2). The number of allocentric based responses was instead particularly high when participants were asked to "do the opposite" of coronal localization $=1$
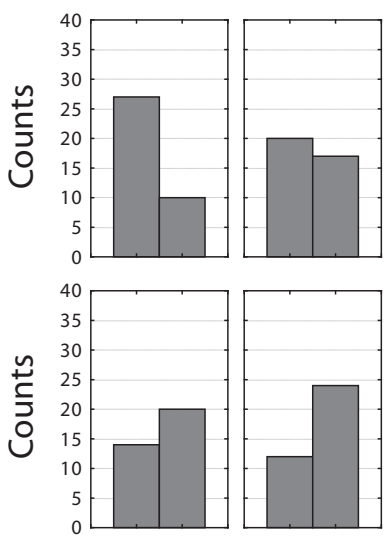

EGO ALLO

gesture: o

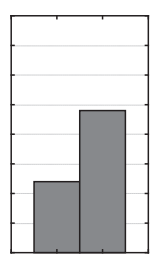

EGO ALLO

gesture: $c$
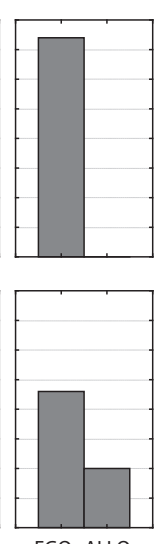

EGO ALLO

gesture: $s$

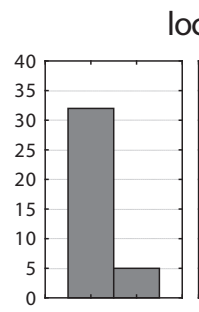

localization $=2$
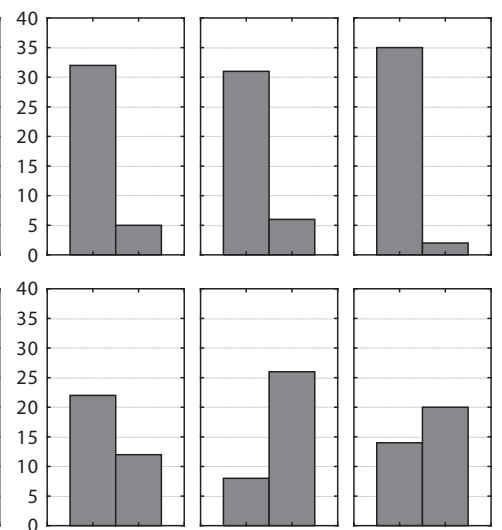

EGO ALLO

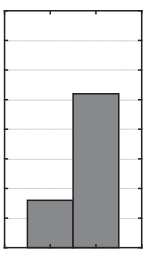

EGO ALLO

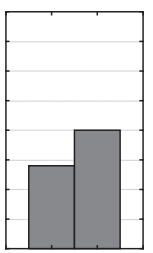

EGO ALLO

gesture: o gesture: $c$ gesture: $\mathrm{s}$
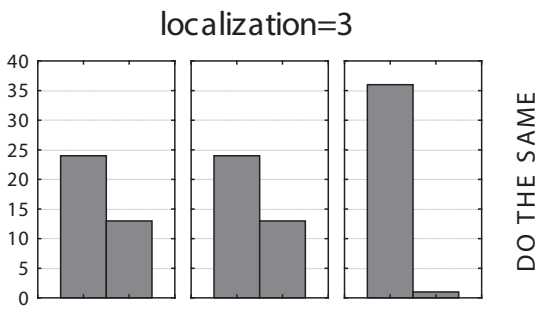

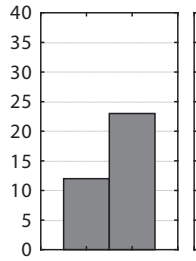

EGO ALLO

gesture: o

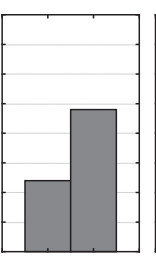

EGO ALLO

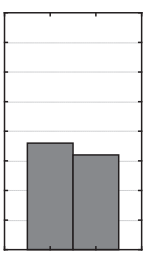

EGO ALLO

gesture: $c$ gesture: $\mathrm{s}$

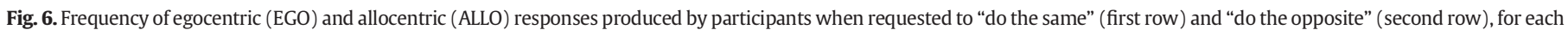
gesture (oscillation, o; coronal gesture, c; sagittal gesture, s) in each localization. 
gestures in all three localizations, and to "do the opposite" of the oscillation gesture when they were localized in front of the model (localization 1) or behind the model (localization 3 ).

\subsubsection{Confidence ratings}

We analyzed whether the confidence of the participants in their responses varied depending on whether they were performing gestures in the counter-directional or homo-directional condition, on their localization with respect to the target, on the type of gesture and on the task (do the same, do the opposite). In order to test this, we conducted a mixed model ANOVA on confidence ratings with orientation (counterdirectional, homo-directional), localization $(1,2,3)$, and gesture (sagittal, coronal, and oscillation) as factors within participants and task as the factor between participants. This analysis revealed that:

a) participants who were asked to "do the same" were not in general any more confident than those asked "to do the opposite", i.e. there was no main effect of task $\left(\mathrm{F}_{(1,66)}=0.794\right.$, $\left.\mathrm{p}=0.376\right)$; in effect, they were on average more than "very confident" in both tasks (mean confidence "do the same" $=3.260$; mean confidence "do the opposite" $=3.147-$ and note that in the scale, 3 corresponded to "very confident" and 4 to "absolutely confident");

b) participants were significantly more confident when they were oriented homo-directionally than counter-directionally (main effect of orientation: $\mathrm{F}_{(1,66)}=67.591, \mathrm{p}<.0001$ ), that is, when the two frames of reference were not in conflict and response satisfied both at the same time;

c) as the significant interaction between gesture and orientation revealed $\left(\mathrm{F}_{(2,132)}=4.192, \mathrm{p}<.02\right)$ in the homo-directional orientation similar confidence ratings were associated with the three gestures (Bonferroni post-hoc, $\mathrm{p}>0.05$ ), while in the counter-directional orientation - where confidence was also significantly lower for all three gestures (Bonferroni post-hoc, $\mathrm{p}<0.001$ ) - participants were more confident with sagittal gestures as compared to coronal gestures (Bonferroni post-hoc, $\mathrm{p}=0.001$ ) or oscillations (Bonferroni post-hoc, $\mathrm{p}<0.001)$;

d) a significant interaction between localization and orientation $\left(F_{(2,132)}=3.299, \mathrm{p}<.05\right)$ also emerged: for all localizations, participants were always more confident when oriented homodirectionally than counter-directionally (Bonferroni, $\mathrm{p}<0.0001$ ) but this difference was slightly smaller for localization 1 as compared to the other localizations.

The main effect of orientation (discussed in point b) proves that participants were more confident when their responses satisfied both frames of reference at the same time (i.e. when orientated homodirectionally). But what happens when gestures are in agreement with only one frame and contrast with the other? To answer this question we calculated for each participant the average confidence of responses based on the egocentric frame, the average confidence of those based on the allocentric frame and the average confidence of those based on both frames simultaneously. We then conducted a mixed effect model ANOVA on these data with type of response as the repeated measure variable, and task as the variable between participants. The results confirmed that task was not significant $\left(F_{(1,51)}=\right.$ $1.561, \mathrm{p}=0.217)$, whereas there was an effect of type of response $\left(F_{(2,102)}=32.024, \mathrm{p}<0.001\right.$; see Fig. 7$)$ : confidence was higher for responses in agreement with both frames, less for responses in agreement only with the egocentric frame and less again for responses in agreement with the allocentric frame (Bonferroni post hoc, $\mathrm{p}<0.02$ ). A tendency toward higher confidence in allocentric responses produced when "doing the opposite" as compared to "doing the same" was also found $(\mathrm{p}=0.07)$.

To sum up, the results from the first study revealed that when oriented counter-directionally and asked to "do the same" as the model, participants rely on the egocentric frame of reference more often than on the allocentric frame. This was confirmed both by the

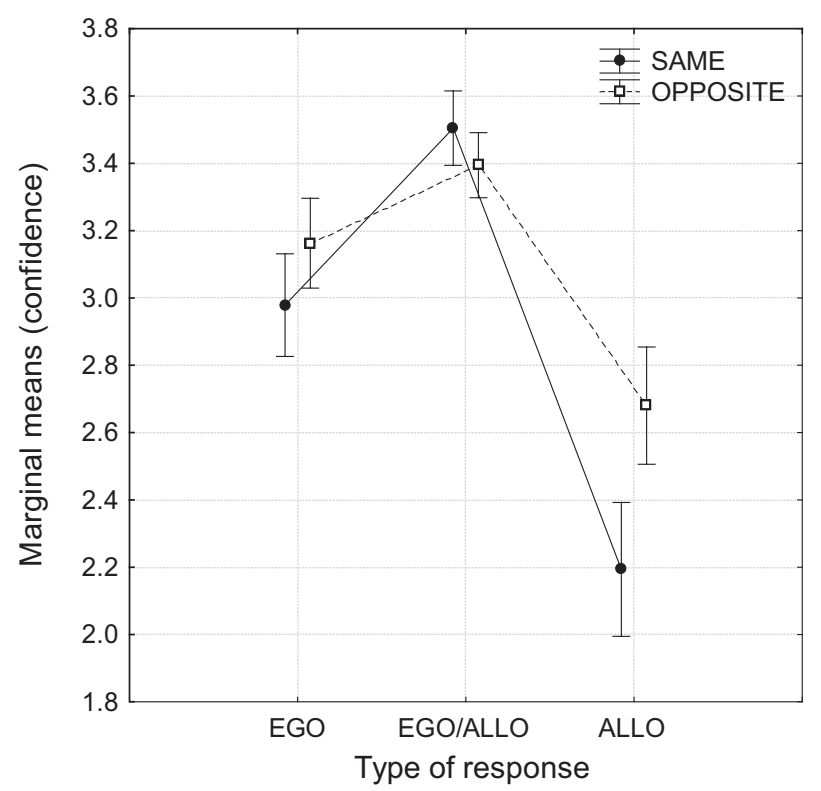

Fig. 7. Mean confidence ratings (and standard errors) associated with responses based on the egocentric frame (EGO), allocentric frame (ALLO) or simultaneously on both frames (EGO/ALLO), in the two tasks (do the same, do the opposite).

overall frequencies and by the proportional preference for egocentric and allocentric responses within participants. This is in agreement with the literature on imitation cited in the Introduction section of this article. Results also showed that egocentric responses were particularly frequent when sagittal gestures were involved.

Conversely, when asked to "do the opposite", participants more frequently relied on environmental coordinates rather than focusing on a body-centered identification of the gestures and this was again confirmed both by the overall frequency and by the proportional preference for egocentric and allocentric responses within participants. Allocentric responses were particularly frequent with coronal gestures, but were also more frequent for the other two types of gestures in specific localizations.

Furthermore, the study showed that "doing the opposite" was not a complicated task or at least participants felt no less confident than when they were requested to imitate a gesture.

Finally, the study revealed that if participants were obliged to base their response on only one of the two spatial frames of reference, they were more confident when relying on the egocentric frame, even though a bias in the opposite direction emerged for "doing the opposite". However when egocentrically-based responses also complied with an environmental definition of identity/opposition (i.e. in the homo-directional orientation), participants were particularly confident of their gestures and this indicates that they were sensitive to both the egocentric and allocentric identities.

\section{Study 2}

In study 2 we used a recognition task rather than a production task, with the aim of verifying whether the indications which had emerged in study 1 would be confirmed. Participants were asked not to actively reproduce a gesture but to describe the relationship perceived when observing two people making gestures. The reasons for this choice are twofold. First, previous studies on "doing the opposite" conducted on visual stimuli revealed that response are not necessarily the same when opposites are produced or recognized (Bianchi \& Savardi, 2008a). 
Second, more in general, in the field of psychophysics, it has been known since 1960 that estimation and production lead to different results. $^{3}$

\subsection{Method}

\subsubsection{Participants}

79 undergraduate students (mean age: 21.3 ) took part in the study.

\subsubsection{Procedure}

The experiment was conducted in a classroom at the University of Macerata. Participants were requested to observe two people (positioned at a distance of $2 \mathrm{~m}$ from each other) performing 12 gestures. The configurations are represented in Fig. 8. Participants were then asked to decide whether the two people were "doing the same" or "doing the opposite" and to assess the degree of evidence of this by means of a 7-point linear bipolar rating scale with "same" and "opposite" at the two extremes of the scale and 0 as the middle point (for cross modal matching methods, see Cascio, 1991; Hayes \& Patterson, 1921). The order of the two poles of the scale (i.e. "same" and "opposite") was counterbalanced between participants. Using a second scale they were next asked to rate how confident they were about their response.

The 12 configurations were presented in random order. The model and the collaborator remained in the position until all the participants had responded. They then performed the next configuration.

\subsubsection{Stimuli}

12 motor configurations were presented to participants (Fig. 8). In all configurations the two people were oriented counter-directionally (i.e. if one was facing north, the other faced south). The bilateral gestures (coronal and sagittal) and localizations (in front of, to the side of, and behind the model) were the same as those used in study 1 . For each gesture made by the model, the second person (the collaborator) performed two gestures: the same gesture from an egocentric frame of reference (which means the opposite allocentrically) and the same gesture from an allocentric frame of reference (which means the opposite egocentrically).

The 12 configurations studied were thus combinations of 3 variables: type of gesture (coronal, and sagittal), localization (in front, to the side, and behind) and structure of the configuration (egocentrically the same/allocentrically the opposite [EGO: s/ALLO: o]; egocentrically the opposite/allocentrically the same [EGO: o/ALLO: s]).

\subsection{Results}

Responses were analyzed with the aim of extracting three pieces of information: A) the frequency of responses based on the egocentric versus allocentric frame of reference; B) evidence of the relationship as expressed by ratings of opposition allocated to configurations with one person making the opposite gesture with respect to the other, and ratings of sameness allocated to configurations with one person doing the same as the other; $\mathrm{C}$ ) confidence in responses.

\footnotetext{
3 The magnitude production procedure results in steeper psychophysical functions than the magnitude estimation procedure - i.e. functions with higher slopes or higher power function exponents. This was found for handgrips (Stevens \& Guirao, 1962; Stevens \& Mack, 1959), loudness (Reynolds \& Stevens, 1960), light and duration (Stevens \& Greenbaum, 1966) and taste (Meiselman, Bose, \& Nikvist, 1972). Stevens and Greenbaum (1966) interpreted this difference between magnitude estimation and magnitude production as a regression effect, wherein the experimental subject tends to constrict the range of the variable dimension. They further suggested that the regression effect is unaffected or little affected by practice, or by the range of stimuli, and that a geometric mean of the two slopes might be the best estimate of the unbiased slope.
}

4.2.1. Frequency of responses based on the egocentric versus allocentric structure of the configuration

A new categorical variable was coded (same, opposite) with the aim of quantifying the number of gestures described as "the same" or "the opposite" based on the egocentric structure of the gestures as compared to those based on the allocentric structure.

Overall participants more often described the gestures according to the egocentric frame (egocentric responses were $70 \%$ of the total number of responses), but were egocentric responses more frequent in general or were they specifically associated with some of the conditions studied? A mixed effect regression model was conducted to test the effects of the type of gesture, the localization and the structure of the configuration on the description of the gesture (i.e. whether it is described as "the same" or "opposite") with same/opposite as a dichotomous dependent variable, structure of the configuration, type of gesture and localization as fixed effects and participants as a random effect. A main effect and two interactions emerged. The main effect of structure of the configuration $\left(\mathrm{F}_{(1,78)}=249.225, \mathrm{p}<0.001\right)$ suggests that participants referred to the egocentric structure more often when the two people were performing egocentrically opposite gestures as compared to egocentrically identical gestures. However, as the interaction between the structure of the configuration and the gesture revealed $\left(F_{(1,78)}=44.216, p<0.001\right)$, this was particularly true when sagittal gestures were involved (i.e. when one person extended their arms in front of them while the other extended them behind) as compared to when coronal gestures were performed (i.e. when one person stretched their arms to the right and the other to their left). The three way interaction between gesture, localization and structure of the configuration $\left(F_{(2,156)}=24.365, \mathrm{p}<0.001\right)$ indicated that this holds for some but not other localizations.

To sum up, a preference for an egocentric based description emerged specifically in 6 of the 12 configurations (see Fig. 9): 4 out of the 6 configurations with sagittal gestures and only 2 out of the 6 configurations with coronal gestures.

With sagittal gestures, when one person extended their arms in front of them while the other extended them behind (Fig. 9, third row), whatever their localization (localizations 1, 2 and 3), they were described as "doing the opposite" (i.e. they referred to the egocentric frame) more frequently than "doing the same" (i.e. they referred to the allocentric frame). However, when both people had their arms stretched out in front of them (Fig. 9, fourth row), only when they were localized one in front of the other (localization 1) were they described by most of the participants as "doing the same" - i.e. participants referred to the egocentric frame; in the other two localizations (localizations 2 and 3), no significant difference was found between the number of responses recognizing the gestures as "the same" (based on the egocentric frame of reference) or "the opposite" (based on the allocentric frame of reference).

With coronal gestures, when the two people were positioned side by side (localization 2) and both extended their arms to their right (Fig. 9, second row), they were described as "doing the same" (i.e. egocentric) significantly more often than as "doing the opposite" (i.e. allocentric); and when the two people were positioned back to back (localization 3 ), one extending his/her arms to the right whereas the other person extended theirs to the left (Fig. 9, first row), they were more often described as "doing the opposite" (i.e. egocentric) rather than "the same" (i.e. allocentric). In the other 4 configurations, the number of responses based on the egocentric frame of reference was not significantly different from the number of responses based on the allocentric frame of reference.

\subsubsection{Ratings of opposition (or sameness) associated with the configurations} perceived as opposite (or same, respectively)

An unbalanced repeated measure ANOVA was conducted on the ratings of opposition for the subset of "opposite" responses with structure of the configuration, gestures and localization as variables within 


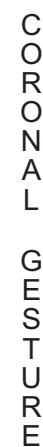

EGO: o / ALLO: $s$<smiles>C=C1CC1</smiles>

(1)
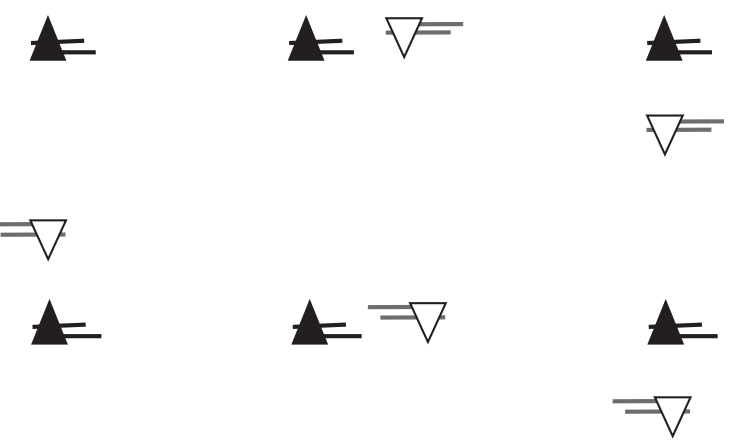

EGO: o / ALLO: s<smiles>C=C1CC1C1CC1</smiles>

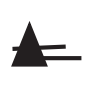

$\boldsymbol{\omega} \forall$

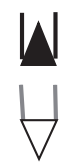

EGO: s / ALLO: o
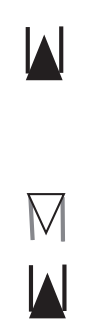

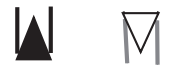

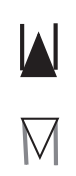

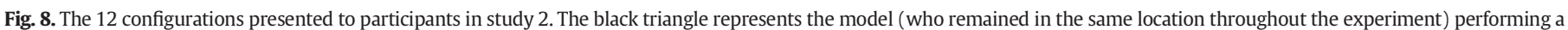

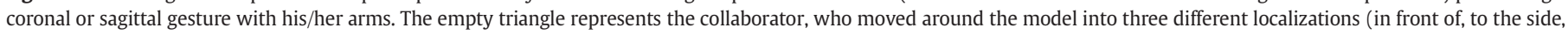

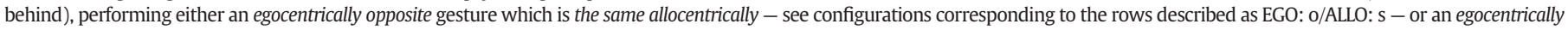
identical gesture which is the opposite allocentrically - see configurations corresponding to the rows described as EGO: s/ALLO: o.

groups. It revealed a main effect of the EGO/ALLO structure of the configuration $\left(\mathrm{F}_{(1,457)}=15.132, \mathrm{p}<.001-\mathrm{Fig} .10\right.$, top-left diagram $)$ which suggested that the degree of opposition recognized in egocentrically opposite gestures was greater than in allocentrically opposite gestures. However, as proved by the interaction between gesture and structure of the configuration $\left(\mathrm{F}_{(1,147)}=14.863, \mathrm{p}<0.001\right)$, this was true only when sagittal gestures were involved (Bonferroni post hoc tests, $\mathrm{p}<0.02$ ) while no differences were found for coronal gestures (see Fig. 10, top-right diagram).

A second unbalanced repeated measure ANOVA was conducted on the ratings of sameness for the subset of "same" responses with structure of the configuration, gestures and localization as variables within groups. Three main effects emerged and no interactions (see Fig. 10, second row). One main effect concerned localization $\left(\mathrm{F}_{(2,310)}=11.924, \mathrm{p}<0.001\right)$ which revealed that ratings were in general higher for those gestures made while the two people were positioned one in front of the other than in the other two localizations (Bonferroni post hoc, $\mathrm{p}<0.02$ ); a second main effect regarded the structure of the configuration $\left(\mathrm{F}_{(1,310)}=\right.$ $6.401, \mathrm{p}<0.001$ ) with higher degrees of "sameness" when the two gestures were the same egocentrically (as compared to allocentrically); a third main effect involved gestures $\left(\mathrm{F}_{(1,310)}=7.690, \mathrm{p}<0.001\right)$ showing that higher ratings of sameness were associated with sagittal gestures. Notice that the penultimate result was not found in previous analyses on the degree of opposition. In other words, egocentric encoding is associated in general with higher ratings of imitation, but not in general with higher ratings of opposition. Ratings of opposition were significantly higher for responses based on the egocentric frame only for sagittal gestures involving one person extending their arms in front while the other extended their arms behind.

\subsubsection{Confidence ratings}

A repeated measure ANOVA on confidence ratings was carried out with type of response, localization, gesture and structure of the configuration as independent variables. No evidence emerged that, in general, the recognition of imitation was more certain than the recognition of opposition (the main effect of the type of response was not significant). Neither were participants more confident when responding according to the egocentric structure of the gesture (the main effect of the structure of the configuration was not significant). As the significant interaction between type of response and localization revealed, participants were more confident when describing the gestures as "the same" in localization 1 , whether allocentrically or egocentrically $\left(\mathrm{F}_{(2,772)}=4.330\right.$; $\mathrm{p}<0.05)$ and when describing the gestures as "opposite" when looking at two egocentrically opposite sagittal gestures, i.e. when one person was moving her arms in front and the other backward (three way interaction between type of response, gesture and structure of the configuration: $\left.\mathrm{F}_{(2,772)}=5.435 ; \mathrm{p}<0.01\right)$.

To sum up, the results of study 2 made it clear that recognition of imitation and opposition between gestures is not strictly limited to the identification of gestures in terms of body coordinates. A generalized use of egocentric encoding might have been expected based on previous literature since the participants were adults and since they were not being asked to respond quickly but had as much time as they wanted. Our results show that the spatial identity of the gestures in terms of 


\section{Localization}

2

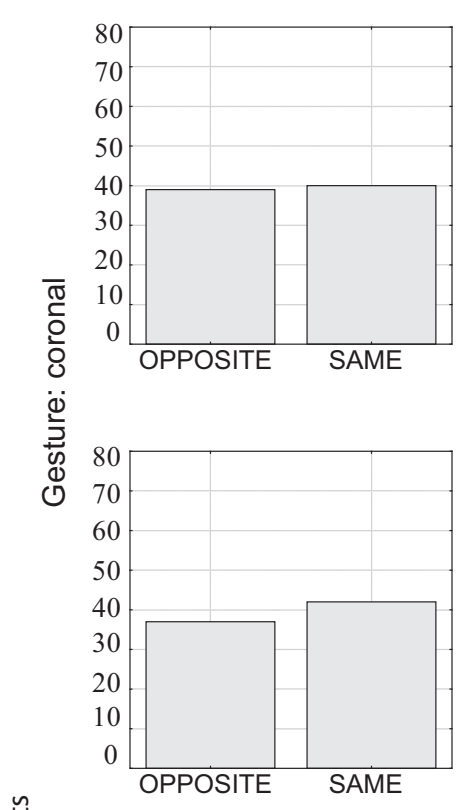

气ั

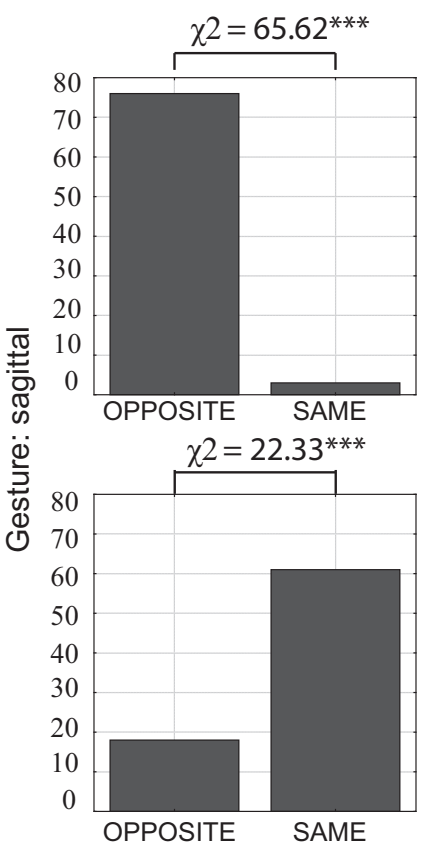

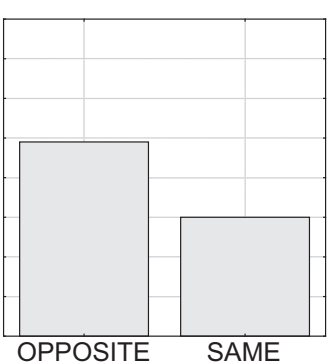
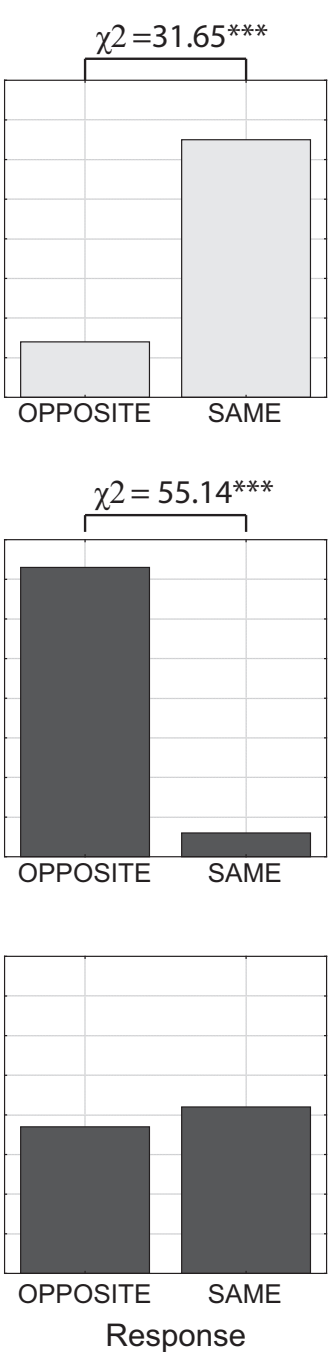
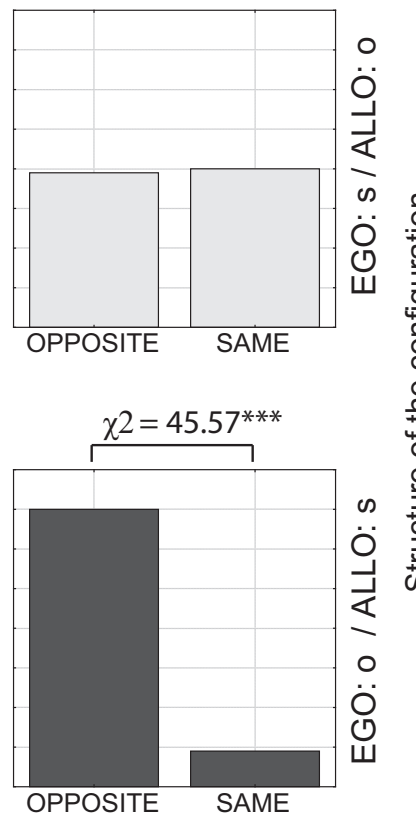

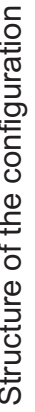

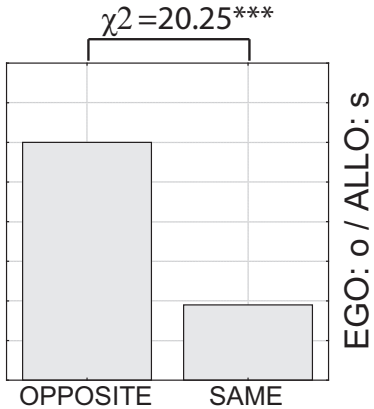

$\infty$

\section{崖}

0

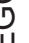

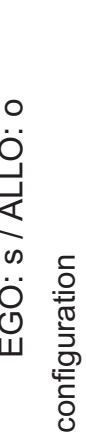

$\stackrel{5}{4}$

$\frac{0}{2}$

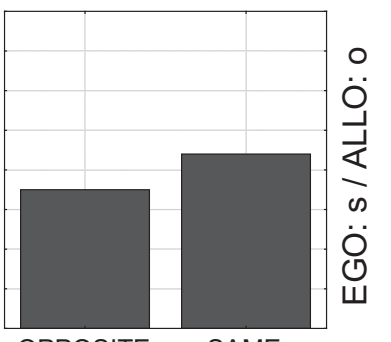

OPPOSITE SAME

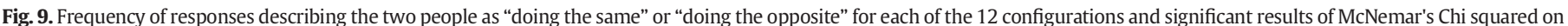
these frequencies $\left(\mathrm{df}=1{ }^{* * *}=\mathrm{p}<0.001\right)$.

allocentric environment in some conditions weakens the "absolute hegemony" of the egocentric frame. Only for the three 'arms straight out in front-arms toward the back' configurations was the contrast between the two gestures in terms of body coordinates too evident to be ignored, whatever the localization of the two people. In this case the participants had no doubts about defining these gestures as "opposite" and were highly confident in doing so. But if we exclude these three configurations, descriptions in terms of body coordinates became less univocal for the other nine, i.e. when both people had their arms straight out in front of the body or both arms stretched out to one side. Only in three of these nine configurations did a significant majority of participants choose an egocentric description of the gesture. For the other six, the identification of the two gestures in terms of the allocentric or egocentric frame turned out to be equiprobable in terms of how frequently participants chose one or the other and there was no difference in terms of evidence and confidence.

\section{Study 3}

In study 3 we went back to a production task, as in study 1 . Gestures involving movements of the whole body in addition to gestures involving movements of only the arms were used in order to test whether the 


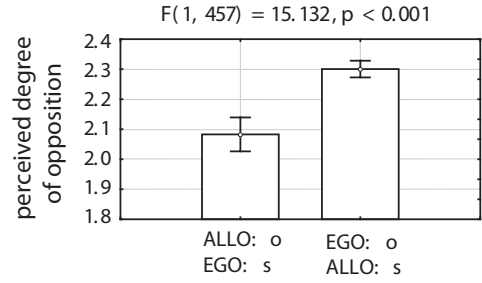

structure of the configuration

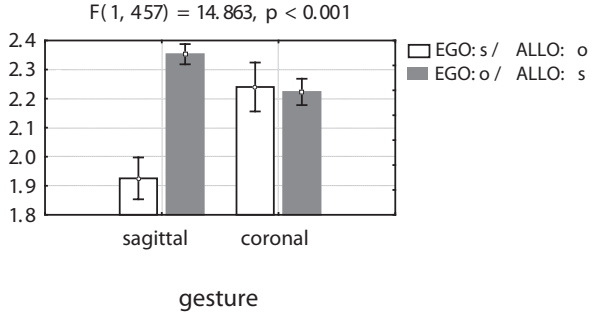

$F(1,310)=7.690, p<0.01$

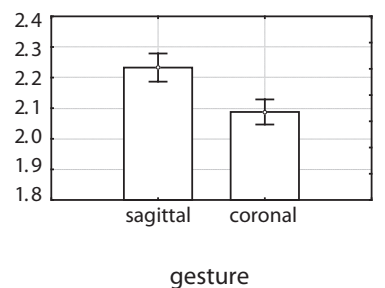

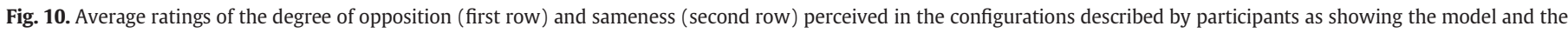
collaborator making, respectively, the same or the opposite gesture. The graphs refer to the main effects and the interactions which turned out to be significant.

salience of the egocentric and allocentric frames of reference changed depending on the local or global nature of the gesture.

Since a preference for the allocentric as compared to the egocentric frame of reference might also depend on whether participants had to "memorize" the gesture (potentially bringing egocentric encoding to the fore) or do it immediately, we studied two different temporal conditions: gesture performed after the target - here "delayed condition" - or at the same time as the target - "simultaneous condition".

In this study we also wished to investigate the immediacy of responses in the two tasks by studying response times. Confidence ratings in study 1 confirmed that participants subjectively considered "doing the opposite" very easy: they were more than "very confident" in their responses. However, if "doing the opposite" is somehow more complicated than "doing the same", this might emerge from response times, that is, longer times should be associated with a more difficult response. Longer times should also be expected if participants, in order to "do the opposite", first imagine "doing the same" and then reverse it.

In order to simplify the experimental design participants were always localized in position 1 and oriented counter-directionally with respect to the model - these in fact turned out to be the most interesting levels of localization and orientation in studies 1 and 2. As in study 1 , the variable task (do the opposite, do the same) was studied between subjects.

\subsection{Method}

\subsubsection{Participants}

80 undergraduate students (mean age: 23.6) participated in the study, divided into four groups: 40 performed the "do the opposite" task (20 in the simultaneous condition, 20 in the delayed condition); 40 performed the "do the same" task ( 20 in the simultaneous condition, 20 in the delayed condition).

\subsubsection{Procedure}

The experiment was conducted in an empty room. Participants took part in the experiment individually. A collaborator of the researcher played the role of the model and performed the gestures, one at a time. The model and the participants occupied the same starting location for the entire duration of the study (they were positioned one in front of the other). The model performed 5 gestures: three gestures using only the arms (local gestures), and two moving the whole body (global gestures). The gestures were performed twice, changing the side of the body (one starting with the left arm/leg, another with the right arm/leg). The order of presentation of the gestures and of the starting side was balanced across participants.

Participants were asked to observe the gesture shown by the model and then (after the model had verbally given the order to start) "do the opposite" or "do the same". In the delayed condition, after the start signal the model remained still while the participants performed his/her response. In the simultaneous condition, after the start signal, the model repeated the gesture so that the participant could perform it at the same time as the model.

Participants were told that there were no right or wrong responses, that they had to give a quick and spontaneous response and that the session was video-recorded to allow researchers to collect response times. After the session was finished they were asked to rate their overall confidence in the responses using the 11-point scale provided which ranged from $0=$ not at all confident to $10=$ totally confident .

\subsubsection{Stimuli}

Ten gestures were presented to each participant in random order ( 5 gestures $\times 2$ starting sides). The 3 local gestures consisted of a sequence of arm movements starting from the same initial position with both arms held close to the body and bent up toward the face. The movements consisted of a) Local Sagittal gesture (LS): the left (or right) arm was stretched out in front of the body and then bent back to return to the initial position, then the other arm was extended down the side of the body and then bent back to return to the initial position; b) Local Coronal gesture (LC): the left (or right) arm was stretched out to the side of the body and bent back to the initial position and then the other arm was extended down the side of the body and bent back to return to the initial position; c) Local Diagonal gesture (LX): the left (or right) arm was stretched out diagonally in front of the body and bent to return to the initial position and then the other arm was extended down the side of the body and bent back to return to the initial position. Two global gestures were also presented. These consisted of Global Sagittal (GS): the model took a step forward starting with the right (or left) leg, repeated the movement with the other leg and then returned to the initial position (moving the right leg and left leg in the same order as that used to step forward); Global Diagonal (GD): the model took a step forward diagonally to the front-left starting with the right leg (or to the front-right starting with the left leg), repeated the movement with the other leg and then stepped back to the initial position using the same order as that used to move forward. 


\subsection{Results}

\subsubsection{Type of response}

Let's consider first of all which characteristics of the initial gesture participants changed when asked to "do the opposite". For both tasks, two independent judges classified whether responses modified the following characteristics of the target gestures or kept them invariant: axis (coronal, sagittal, or diagonal), direction within the axis (e.g. front-back, up-down), shape of the gesture and temporal order of the phases of the gesture (the latter was considered only for local gestures, which consisted of a movement in two phases and thus it would be possible to "do the opposite" by reversing the temporal order of the two phases). The inter-rater agreement was excellent (Cohen's $\kappa=0.89$ ). An analysis of the frequency of these categories or responses showed that in the imitation task, the great majority of participants kept the axis (99\%), the direction (94\%), the shape (98\%) and the temporal order (97\%) invariant with respect to the model. This is an obvious result. But even when asked to "do the opposite", participants very rarely modified the shape of the gesture ( $1 \%$ in the simultaneous condition, $3 \%$ in the delayed condition), or altered its axis ( $1 \%$ in both conditions). They sometimes reversed the temporal phases of the gestures ( $8 \%$ in the simultaneous condition, $22 \%$ in the delayed condition). In the majority of responses ( $84 \%$ in the delayed condition, $90 \%$ in the simultaneous condition) they inverted the spatial direction of the gesture. But in terms of which frame of reference? In other words, did participants decide to "do the opposite" or "the same" based on the allocentric or egocentric space? Since for every gesture responses could be either based on one or the other frame, we tested whether the proportion of responses based on one or the other was significantly different from chance using binomial tests (probability test proportion $=0.50$ ).

In general, independently of the task, allocentric responses were more frequent $(\mathrm{N}=641)$ than egocentric responses $(\mathrm{N}=219)$ (observed proportions: $\mathrm{ALLO}=0.75 \mathrm{vs}$. $\mathrm{EGO}=0.25, \mathrm{p}<0.001$ ). This was confirmed for both tasks (observed proportion in the "do the same" task: $\mathrm{ALLO}=0.81 \mathrm{vs}$. EGO $=0.19, \mathrm{p}<0.001$; observed proportion in the "do the opposite" task: ALLO $=0.68$ vs. $\mathrm{EGO}=0.32, \mathrm{p}<0.001)$ and in both the delayed and simultaneous conditions (observed proportions in simultaneous imitation: $\mathrm{ALLO}=0.76$ vs. $\mathrm{EGO}=0.24, \mathrm{p}<0.001$; in delayed imitation: $A L L O=0.86$ vs. $E G O=0.14, p<0.001$; in simultaneous opposition: $\mathrm{ALLO}=0.72$ vs. $\mathrm{EGO}=0.28, \mathrm{p}<0.001$; in delayed opposition ALLO $=0.63$ vs. $\mathrm{EGO}=0.37, \mathrm{p}<0.001$ ).

The results of a mixed effect regression model on the type of response dichotomous variable (EGO, ALLO), with task (do the same, do the opposite) and condition (delayed, simultaneous) as fixed effects and participants as a random effect (nested in task) revealed the main effect of task $\left(\mathrm{F}_{(1,856)}=5.406, \mathrm{p}=0.02\right)$ indicating that the proportion of allocentric responses was higher when people were "doing the same".

\subsubsection{Response times}

Participants' response times were measured by two independent judges from the beginning of the vocal "start" signal (given by the model after executing the gesture) to the culmination of the movement performed by the participant. ${ }^{4}$ Inter-rater agreement turned out to be excellent (ICC $=0.975 ; 0.971<95 \%$ confidence interval $<0.978$ ). The following analyses were performed on a data matrix obtained by averaging the response times measured by the two raters.

Plots of average response times (Fig. 11) allowed us to explore whether response times varied depending on the temporal condition (simultaneous, delayed), the frame of reference (EGO, ALLO), the

\footnotetext{
${ }^{4}$ The culmination point was considered to be the full extension of the arms for local arm gestures (i.e. coronal and sagittal gestures) and the point of maximum displacement of the body for global gestures.
}

gesture (LC, LX, LS, GC, GX), the starting side (left, right) and the task (do the same, do the opposite). Interesting differences seemed to emerge. In order to test these differences statistically and to study any possible interaction between variables, we planned to conduct a mixed model ANOVA with gesture and starting side as within subject variables and all other factors between subjects. A preliminary exploration of the distribution of response times revealed however that data did not match the requisite of normality (see the diagrams on the left in Fig. 12). To check what an appropriate normalization transformation of the raw response times might be, we used Box and Cox (1964) procedure which suggested that $|1 / \mathrm{x}|$ was the transformation which guaranteed a fit with a normal distribution (see Fig. 12, diagrams on the right). We thus applied this transformation to our raw data and then conducted a linear mixed-effect model ANOVA on these data (we will refer to them as RT).

Various significant main effects emerged:

a) Temporal Condition $\left(\mathrm{F}_{(1,955)}=85.859, \mathrm{p}<0.001\right)$ : in the delayed condition participants took significantly more time before responding than in the simultaneous condition (RT delayed $>$ RT simultaneous);

b) Frame of Reference $\left(\mathrm{F}_{(1,955)}=10.388, \mathrm{p}<0.001\right)$ : allocentric responses were faster than egocentric responses (RT ego > RT allo);

c) Task $\left(\mathrm{F}_{(1,955)}=5.424, \mathrm{p}=0.02\right)$ : participants were faster when "doing the opposite" than when imitating (RT do the same $>$ RT do the opposite);

d) Gesture $\left(\mathrm{F}_{(4,347)}=4.956, \mathrm{p}<0.001\right)$ : participants took longer to respond to sagittal gestures as compared to (local or global) gestures involving a coronal or diagonal direction (RT LS > RT LC, LX, Bonferroni post hoc, $\mathrm{p}<.001$; RT LS $>$ RT GC, Bonferroni post hoc, $\mathrm{p}<0.02)$.

e) Starting side $\left(\mathrm{F}_{(1,955)}=3.393, \mathrm{p}=0.06\right)$ : participants tended to be slower when the gesture performed by the model started with the left arm/leg (RT starting left $>$ RT starting right).

Various significant interactions also emerged, which assisted us to interpret these results better:

- Temporal condition $*$ Frame $\left(\mathrm{F}_{(1,955)}=12.950, \mathrm{p}<0.001\right)$ : when participants produced their gestures concurrently with the model, no significant differences emerged between responses based on one or the other frame. It was in the delayed condition that egocentric responses were slower than allocentric responses (RT Delayed_EGO > RT Delayed_ALLO, p $<0.001)$. Allocentric responses were faster both when participants were "doing the same" and when they were "doing the opposite" (RT Delayed_EGO_s > RT Delayed_ALLO_s, $\mathrm{p}<$.001; RT Delayed_EGO_o $>$ RT Delayed_ALLO_0, $\mathrm{p}<.02)$.

- The interaction between Temporal condition $*$ gesture $\left(\mathrm{F}_{(4,374)}=\right.$ $4.113, p=0.003)$ revealed that in the delayed condition, responses for sagittal gestures were slower than any other local or global gestures (RT Delayed_LS $>$ RT Delayed_LC,LX,GC,GX, p $<0.01$ ). In the simultaneous condition, there was also a difference between local coronal or diagonal gestures and their corresponding global gestures, with the local gestures faster than the global ones (RT Simultaneous_GC,GX $>$ RT Simultaneous_LC,LX p $<0.01$ ).

- The interaction between Temporal conditions $*$ Frame $*$ Gesture $*$ Task $\left(\mathrm{F}_{(4,374)}=2.488, \mathrm{p}<0.05\right)$ revealed that, although response times varied depending on the gesture, when there were differences between response times associated with the two tasks participants were always quicker to "do the opposite" than "the same", both allocentrically and egocentrically. This difference between tasks was found in three cases in the delayed condition: allocentric responses to global coronal gestures (GC: RT Delayed_ALLO_o $<$ RT Delayed_ALLO_s, p $<0.05)$ and with egocentric responses to local coronal and diagonal gestures (LC, LX: RT Delayed_EGO_o $<$ RT Delayed_EGO_s, p < 0.05). In the simultaneous condition, a similar bias was found for local sagittal responses (LS: RT 

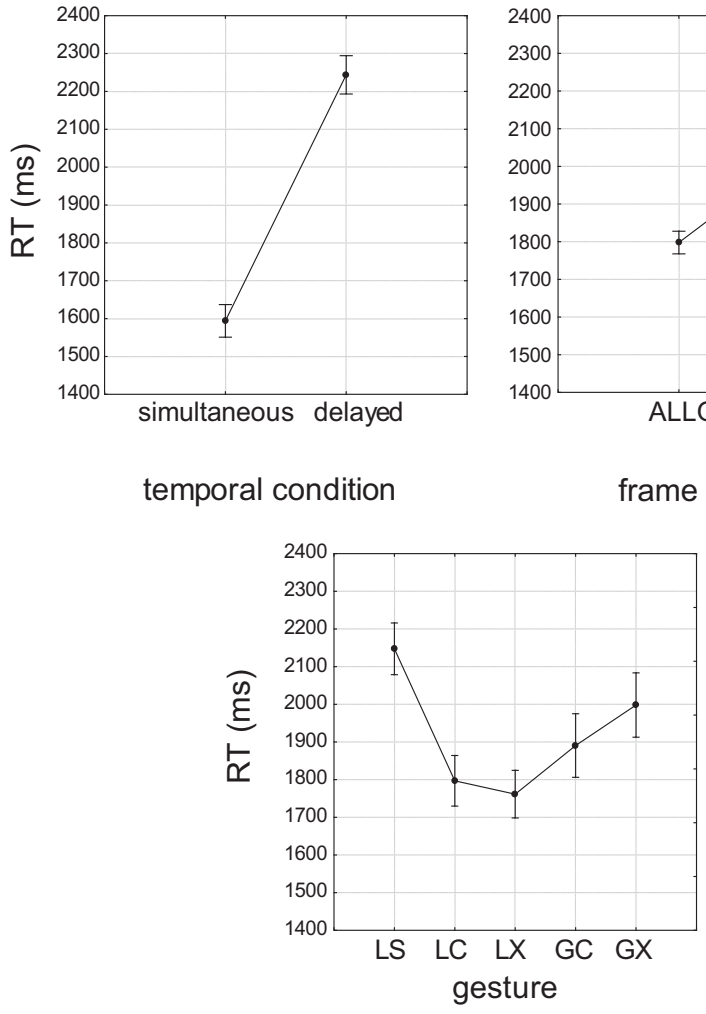

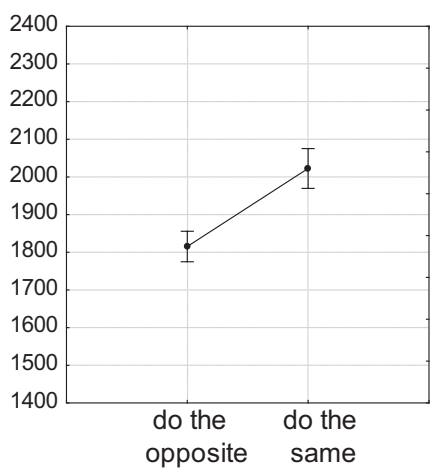

task

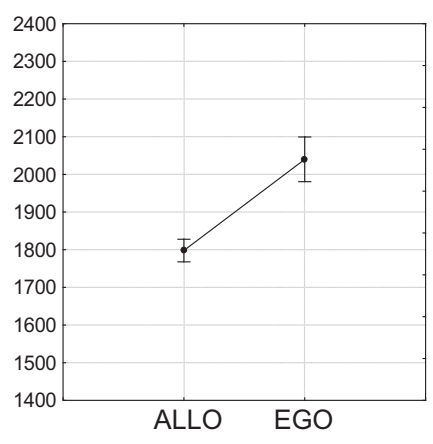

frame of reference

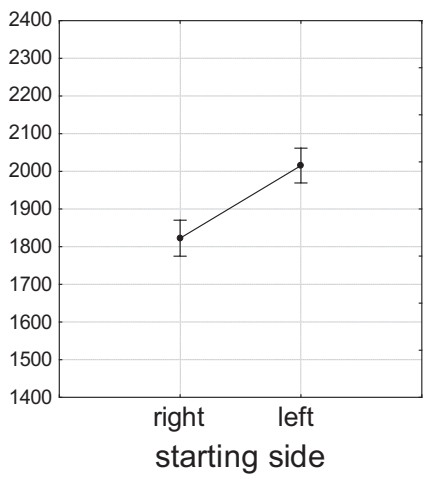

Fig. 11. Average response times (and standard errors) associated with the different levels of the independent variables analyzed in study 3.

Simultaneous_ALLO_o $<$ RT Simultaneous_ALLO_s, p $=0.06)$.

- Temporal condition $*$ Frame $*$ Starting side $*$ Task $\left(\mathrm{F}_{(1,955)}=4.040\right.$, $\mathrm{p}<0.05)$ : for imitations based on the egocentric frame of reference, slower response times were found when the target gesture performed by the model started with the left arm/leg (which means that participants - in order to imitate it egocentrically
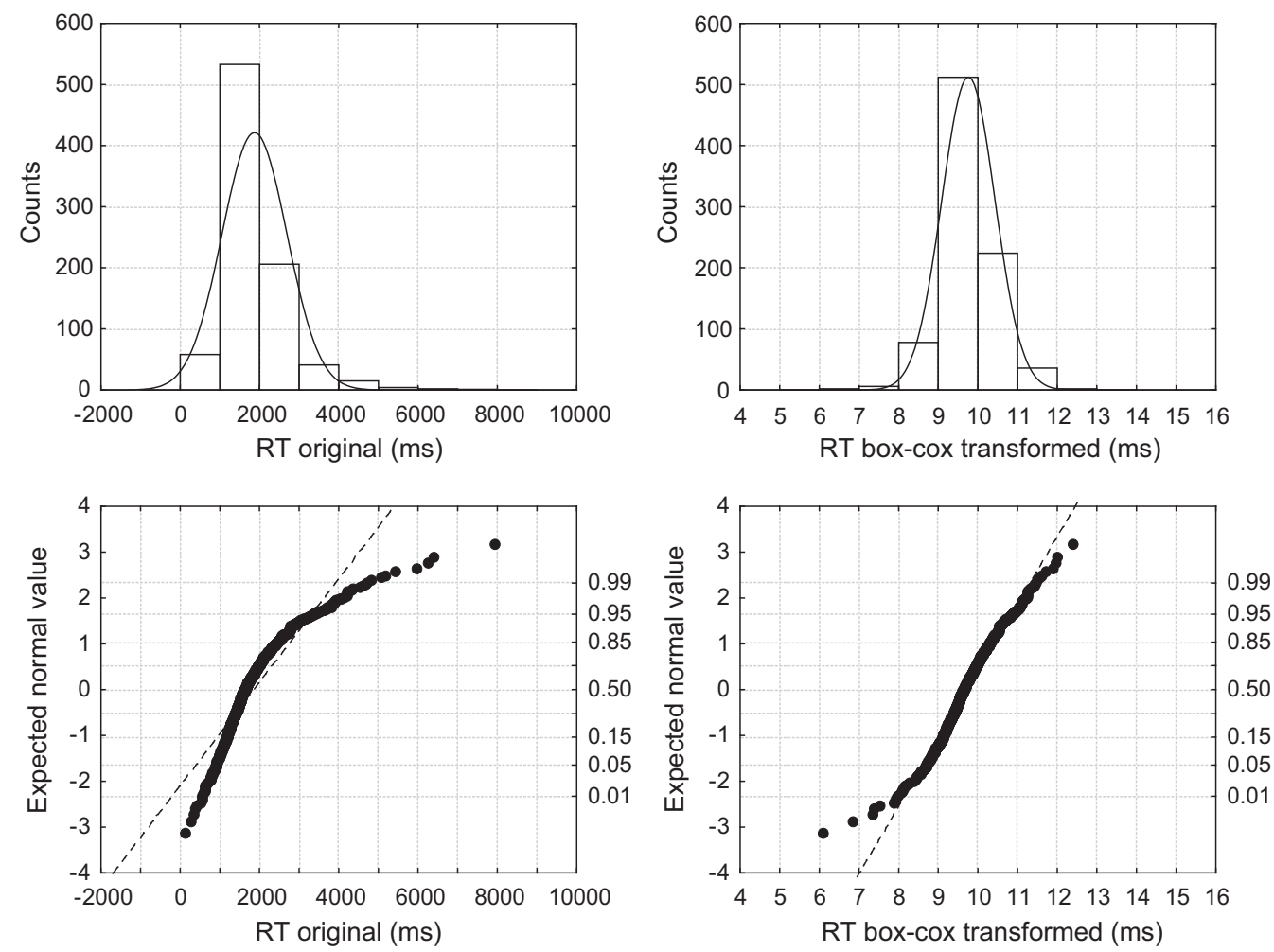

Fig. 12. Histograms (diagrams above) and normal probability plots (diagrams below) of raw response times. 
- had to also move their left arm/leg). With only two exceptions, our participants were right-handed and this could explain why they found it easier to respond egocentrically to right-sided gestures than to leftsided gestures.

\subsubsection{Confidence rating}

Lastly, we tested whether the confidence expressed by participants at the end of their experimental session varied according to the temporal conditions (delayed, simultaneous), the task (do the same, do the opposite), the proportion of egocentric and allocentric responses given by each participant as part of the total number of responses to the 10 stimuli and the mean response time (this latter was calculated by averaging, for each participant, the response times of the 10 gestures that he/she performed). An ANOVA was conducted on confidence ratings, with temporal condition and task as independent category variables and mean response time and frequency of EGO and ALLO responses as covariates. Only a trend regarding the main effect of response times emerged $\left(F_{(1,80)}=3.608, p=0.06\right)$. An inverse correlation between confidence ratings and mean response times was found (Kendall $\tau=$ $-0.21, p=0.003$ ), i.e. shorter average response times were associated with higher confidence.

\section{Final discussion}

The aim of the studies presented in this paper is to contribute to a better understanding of the roles that the system of coordinates of a person's body (the egocentric frame of reference) and the environmental system of coordinates (the allocentric frame of reference) play in the spatial organization underlying the recognition and production of imitational and oppositional gestures. We propose that new insights might arise if we consider the structure of gestures perceived as opposite instead of exclusively focusing on imitation - until now the most common perspective in the literature. The first reason for this is that the egocentric and allocentric frames of reference which are essential to any experience of space have an intrinsic oppositional structure (in front-behind, left-right and up-down, north-south, east-west, and above-below). Furthermore human perception of space is modeled by basic structures of contrariety (Bianchi \& Bertamini, 2013; Bianchi, Savardi, \& Burro, 2011; Bianchi, Savardi, \& Kubovy, 2011; Bianchi et al., 2013; Savardi \& Bianchi, 2009) both when it concerns the perceived extension of objects and environments (e.g. high-low, largesmall, broad-narrow, long-short, near-far ...), and their orientation (i.e. vertical-horizontal, convergent-divergent, downhill-uphill ...), and when position (e.g. above-below, top-bottom, beginning-end, lying-suspended ...) and form (e.g. regular-irregular, symmetricasymmetric, straight-curved, angular-rounded ...) are involved. There is also evidence that spatial oppositions are primal in human development (Casasola, 2008; Casasola et al., 2003; Hespos \& Spelke, 2004; McDonough, Choi, \& Mandler, 2003; Quinn \& Bhatt, 2005; Quinn, Cummins, Kase, Martin, \& Weisman, 1996). Furthermore, “doing the opposite" is something which occurs in daily gestures much more frequently than one might expect, as we pointed out in the Introduction section.

For all these reasons we did not expect opposition tasks to be convoluted. And indeed, as the results from study 1 confirmed, participants in the study estimated themselves to be more than very confident when "doing the opposite"; they felt no less confident than when they were requested to imitate a gesture. In addition, as the analysis of response times in study 3 confirmed, adults were often quicker when "doing the opposite" than when "doing the same". These data are intriguing and lead us to think that we should not underestimate the difficulty of imitating and at the same time not overestimate the difficulty of "doing the opposite".

A comparison between the two tasks and three studies allowed us to understand the importance of the two frames of reference when people did, respectively, "the same" and "the opposite". A direct generalization from literature on imitation might have led one to predict, on the one hand, that although children would likely rely on the allocentric system to solve the tasks, this solution would be progressively abandoned with age and from 18 years - according to Wapner and Cirillo's results on imitation (1968) - 80-85\% of adults would base their responses on the egocentric frame. On the other hand, there have been behavioral studies showing that when adults are rotated through $180^{\circ}$ they are more accurate and quicker at imitating non-anatomically (Avikainen et al., 2003; Franz et al., 2007) which leads us to suppose that the allocentric system might still play a role in adults. The interpretation of the preference for non-anatomical matching at $180^{\circ}$ in terms of left-right spatial compatibility (Heyes \& Ray, 2004) is not in disagreement with the idea that the allocentric frame of reference is involved here. The fact that actions are executed faster and more accurately in response to stimuli that are in the same left-right spatial location as the response hand or which appear to move toward the response hand (Bosbach, Prinz, \& Kerzel, 2004; Cooper, Catmur, \& Heyes, 2013; Proctor \& Vu, 2006; Umiltà \& Nicoletti, 1990) is compatible with saying that responses are faster and more accurate when the same part of space from an allocentric point of view is involved.

In general, two main results emerge from our studies with regard to the use of the allocentric and egocentric frames of reference in the two different tasks. Firstly, the findings from our studies provide multiple indications that both frames of reference count (on the interplay between the two, see also Kaplan, 2013). A first indication that both frames are used comes from confidence ratings in study 1. Participants were less certain about their response when the gesture performed complied with only one frame and contrasted with the other (and this was the rule when the two people involved were counter-directionally oriented), as compared to when both frames were consistent with each other (i.e. homo-directional orientation). If the criterion followed by adults was simply that of producing an egocentric response, then they would have no reason to feel that certain responses (i.e. those in agreement also with the allocentric frame) were more convincing that others once the match in terms of body coordinates was guaranteed.

A second indication is demonstrated by the frequency of responses based on the egocentric frame. In both studies 1 and 2 this frame of reference is more frequently referred to, but the allocentric frame is preferred in study 3 . In addition, again in studies 1 and 2, the percentage of responses that the egocentric frame can account for is not always high enough to justify the supposition that the allocentric frame is irrelevant. In study 1, the allocentric frame was used in only $20 \%$ of responses by the group of participants who were asked to "do the same" as the model, but in 54\% of responses from those asked "do the opposite". In study 2 , in only 6 out of the 12 configurations were responses more frequently based on the egocentric frame; in the remaining 6 configurations, responses were divided equally between egocentric and allocentric. Moreover, as already mentioned, when participants were prompted to respond quickly (study 3 ) the allocentric frame of reference became prominent for both "doing the same" and "the opposite".

A third source of evidence that responses were not simply based on bodily coordinates but on a more complex spatial structure involving the two frames is that significant interactions were found involving gestures and localizations. This proves that participants were not systematically (and blindly) applying one or the other frame independently of whether the gestures were sagittal or coronal or diagonal or independently of the reciprocal localization of the individuals. The visual structure of the configuration evidently influenced their responses.

The second main result emerging from our studies is that, in adults, the role of the allocentric system in spatial organization can be revealed by requesting someone to quickly imitate a gesture (as in study 3 ). In study 3 allocentric responses were more frequent for gestures involving "doing the same" than for those involving "doing the opposite". However, the allocentric frame of reference is more consistently linked to opposite gestures in the sense that there is a more robust association 
between "doing the opposite" and doing it allocentrically and people are less sensitive to temporal conditions (i.e. quick responses or free responses in terms of the time taken to respond). In fact, the temporal condition is absolutely critical in imitation tasks when the allocentric frame of reference is only used more often than the egocentric frame of reference if people have to respond quickly, otherwise the majority of them choose to imitate egocentrically. However, when they are asked to produce an opposite gesture, people often (as in study 1 ) or always (as in study 3 ) rely on the allocentric frame of reference more frequently than the egocentric frame, independently of whether the task leaves them free to respond at their own pace (study 1 ) or obliges them to give a quick response (study 3 ).

In numerous theoretical perspectives it has been proposed that independent representations, coordinate systems, and/or processing modules - labeled from time to time as relative and absolute (Schmidt, 1975), visual/spatial and motor (Bapi, Doya, \& Harner, 2000; Chaminade et al., 2005), cognitive and motor (Keele, Jennings, Jones, Caulton, \& Cohen, 1995; Verwey, 1999) - contribute to the coding and production of gestures. Our results are in agreement with the experimental evidence which has emerged in the last decade from studies on the learning of movement sequences, supporting the importance of the role of the allocentric visual-spatial coordinates versus the egocentric effector-dependent codes (Bapi et al., 2000; Bird \& Heyes, 2005; Boutin, Fries, Panzer, Shea, \& Blandin, 2010; Heyes \& Foster, 2002; Hikosaka et al., 1999, 2002; Kovacs, Muehlbauer, \& Shea, 2009; Osman, Bird, \& Heyes, 2005).

Our findings are also in line with a significant result from an investigation into the relationship perceived by adults when they look at their reflections in a mirror (Bianchi \& Savardi, 2008c, 2009; Savardi, Bianchi, \& Bertamini, 2010). This demonstrated that, contrary to the emphasis on the egocentric frame of reference in the literature focusing on leftright reversal (e.g. Corballis, 2000; Gregory, 1996; Haig, 1993; Navon, 1987; Takano, 1998), the allocentric relationship perceived between the orientation of the body and of its reflection strongly affects the direct perception of reflections. Since some of the configurations studied in this paper may have called to mind mirror patterns (either the orientation of the two bodies and/or the position of the arms), in conclusion, we would like to take time to consider this point.

In study 1 , in the counter-directional orientation, the patterns produced when participants were asked to "do the opposite" were in most of the cases allocentrically opposite for coronal and oscillatory gestures, which corresponds to the opposite of how a reflection in a mirror behaves. But for sagittal gestures, participants who were asked to "do the opposite" produced (in a non-significantly different percentage of cases) a gesture in agreement with what a mirror would reflect (i.e. model: arms extended forward - participant: arms extended forward) or a response which was opposite to what the mirror would have shown (i.e. model: arms extended forward - participant: arms extended behind his/her back). In study 2, there were four configurations which were compatible with reflection patterns, i.e. model with arms extended to the right with the collaborator extending his/her arms to the left in localizations 1 and 3 and both model and collaborator with their arms extending forward in localizations 1 and 3. Three of these four configurations were described as being "the same" or as being "opposite" with no significant differences. These responses indicate that participants are not consistently assuming the "mirror pattern" as an example of identity or opposition. They seem rather confused. But these responses are indeed consistent with the ambiguous relationship perceived by adults when they look at reflections of their own body and of other peoples' bodies in large wall mirrors (Bianchi and Savardi, 2008c). Reflections on a single plane mirror set vertically on the wall in front of an observer were usually described as being identical to the original body ( $90 \%$ of responses). However, they were at the same time recognized as having an opposite orientation (80\%), mainly owing to the opposite sagittal allocentric orientation and only to a lesser extent (less than 30\%) to the recognition of egocentric left-right reversal. An analysis of the responses produced when people were asked to perform simple gestures such as those studied in the present paper and to describe how they perceived the reflected gesture with respect to that made by themselves confirmed that, in general, the gesture was perceived as "opposite" in configurations with the observer and his/her reflection pointing in opposite allocentric directions and "the same" when the reflected gesture pointed in the same allocentric direction (without paying too much attention to which arm was being used). This ambiguous perception of the spatial orientation of reflections in terms of "doing the same" and/or "doing the opposite" is so robust that it can explain some kinds of errors made by adults when they predict the reflected movement which would correspond to a given real movement (Savardi et al., 2010). Further research will prove if, as we are inclined to believe, the investigation of how the oppositional structure of space impacts on human perception and performance is in fact an issue which has been underestimated.

\section{Acknowledgments}

We would like to thank the three anonymous reviewers for their valuable comments and Michael Kubovy for his precious advice regarding methodology.

\section{References}

Avikainen, S., Wohlschläger, A., Liuhanen, S., Hanninen, R., \& Hari, R. (2003). Impaired mirror-image imitation in Asperger and high-functioning autistic subjects. Current Biology, 13, 339-341.

Bapi, R. S., Doya, K., \& Harner, A.M. (2000). Evidence for effector independent and dependent representations and their differential time course of acquisition during motor sequence learning. Experimental Brain Research, 132, 149-162.

Bekkering, H., Wohlschlager, A., \& Gattis, M. (2000). Imitation of gestures in children is goal-directed. Quarterly Journal of Experimental Psychology, A, 53, 153-164.

Berges, J., \& Lezine, I. (1963). Test d'Imitation de Gestes: Techniques d'Exploration du Schema Corporel et des Praxies Chez l'Enfant de 3 A 6 Ans. Paris, France: Masson and Cie.

Bianchi, I., \& Bertamini, M. (2013). Anisotropy and polarization of space: Evidence from naïve optics and phenomenological psychophysics. Behavioral and Brain Sciences, 36(5), 545-546

Bianchi, I., Burro, R., Torquati, S., \& Savardi, U. (2013). The middle of the road: Perceiving intermediates. Acta Psychologica, 144(1), 121-135.

Bianchi, I., \& Savardi, U. (2008a). The perception of contraries. Roma, Italy: Aracne.

Bianchi, I., \& Savardi, U. (2008b). Contrariety as perceptual relationship. Gestalt Theory, 2, $106-120$.

Bianchi, I., \& Savardi, U. (2008c). The relationship perceived between the real body and the mirror image. Perception, 5, 666-687.

Bianchi, I., \& Savardi, U. (2009). Contrariety in plane mirror reflections. In U. Savardi (Ed.) The perception and cognition of contraries (pp. 113-128). Milano, Italy: Mc-Graw Hill.

Bianchi, I., Savardi, U., \& Burro, R. (2011). Perceptual ratings of opposite spatial properties: Do they lie on the same dimension? Acta Psychologica, 138(3), 405-418.

Bianchi, I., Savardi, U., \& Kubovy, M. (2011). Dimensions and their poles: A metric and topological theory of opposites. Language and Cognitive Processes, 26(8), 1232-1265.

Binkofski, F., Amunts, K., Stephan, K. M., Posse, S., Schormann, T., Freund, H. -J., et al. (2000). Broca's region subserves imagery of motion: A combined cytoarchitectonic and fMRI study. Human Brain Mapping, 11, 273-285.

Bird, G., \& Heyes, C. (2005). Effector-dependent learning by observation of a finger movement sequence. Journal of Experimental Psychology: Human Perception and Performance, 31, 262-275.

Bosbach, S. Prinz W., \& Kerzel, D. (2004). A Simon effect with stationary moving stimuli. Journal of Experimental Psychology: Human Perception and Performance, 30, 39-55.

Boutin, A., Fries, U., Panzer, S., Shea, C. H., \& Blandin, Y. (2010). Role of action observation and action in sequence learning and coding. Acta Psychologica, 135(2), 240-251.

Box, G. E. P., \& Cox, D. R. (1964). An analysis of transformations (with discussion). Journal of the Royal Statistical Society: Series B: Methodological, 26, 211-252.

Buccino, G., Binkofski, F., Fink, G. R., Fadiga, L., Fogassi, L., Gallese, V., et al. (2001). Action observation activates premotor and parietal areas in a somatotopic manner: An fMR study. European Journal of Neuroscience, 13, 400-404.

Byrne, P., \& Becker, S. (2008). A principle for learning egocentric-allocentric transformation Neural Computation, 20(3), 709-737.

Casasola, M. (2008). The development of infants' spatial categories. Current Directions in Psychological Science, 17, 21-25.

Casasola, M., Cohen, L. B., \& Chiarello, E. (2003). Six-month-old infants' categorization of containment spatial relations. Child Development, 74, 679-693.

Cascio, W. F. (1991). Applied psychology in personnel management (4th ed.). Englewood Cliffs, NJ: Prentice-Hall.

Chaminade, T., Meltzoff, A. N., \& Decety, J. (2005). An fMRI study of imitation: Action representation and body schema. Neuropsychologia, 43, 115-127.

Chiavarino, C., Apperly, I. A., \& Humphreys, G. W. (2007). Exploring the functional and anatomical bases of mirror-image and anatomical imitation: The role of the frontal lobes. Neuropsychologia, 45(4), 784-795. 
Cooper, R. P., Catmur, C., \& Heyes, C. (2013). Are automatic imitation and spatial compatibility mediated by different processes? Cognitive Science, 37(4), 605-630.

Corballis, M. C. (2000). Much ado about mirrors. Psychonomic Bulletin E Review, 7. 163-169.

Decety, J., Chaminade, T., Grezes, J., \& Meltzoff, A. N. (2002). A PET exploration of the neural mechanisms involved in reciprocal imitation. Neurolmage, 15, 265-272.

di Pellegrino, G., Fadiga, L., Fogassi, L., Gallese, V., \& Rizzolatti, G. (1992). Understanding motor events: A neurophysiological study. Experimental Brain Research, 91, 176-180.

Fogassi, L., Gallese, V., Fadiga, L., \& Rizzolatti, G. (1998). Neurons responding to the sight of goal-directed hand/arm actions in the parietal area PF (7b) of the macaque monkey. Society for Neuroscience - Abstracts, 24, 654.

Franz, E. A., Ford, S., \& Werner, S. (2007). Brain and cognitive processes of imitation in bimanual situations: Making inferences about mirror neuron systems. Brain Research, 1145, 138-149.

Gallese, V., Fadiga, L., Fogassi, L., \& Rizzolatti, G. (1996). Action recognition in the premotor cortex. Brain, 119, 593-609.

Genschow, O., Florack, A., \& Wänke, M. (2013). The power of movement: Evidence for context-independent movement imitation. Journal of Experimental Psychology: General, 142(3), 763-773.

Gleissner, B., Meltzoff, A. N., \& Bekkering, H. (2000). Children's coding of human action: Cognitive factors influencing imitation in 3-year-olds. Developmental Science, 3(4), 405-414.

Grafton, S. T., Arbib, M.A., Fadiga, L., \& Rizzolatti, G. (1996). Localization of grasp representations in humans by positron emission tomography. II. Observation compared with imagination. Experimental Brain Research, 112, 103-111.

Gregory, R. L. (1996). Mirrors in mind. New York: Freeman Spektrum.

Grezes, J., Costes, N., \& Decety, J. (1999). The effects of learning and intention on the neural network involved in the perception of meaningless actions. Brain, 122, $1875-1887$.

Haig, N. D. (1993). Reflections on inversion and reversion. Perception, 22, 863-868.

Hayes, M. H., \& Patterson, D.G. (1921). Experimental development of the graphic rating method. Psychological Bulletin, 18, 98-99.

Hespos, S. J., \& Spelke, E. S. (2004, July). Conceptual precursors to language. Nature, 430(6998), 453-456

Heyes, C. M. (2001). Causes and consequences of imitation. Trends in Cognitive Sciences, 5 , $253-261$.

Heyes, C. M. (2011). Automatic imitation. Psychological Bulletin, 137(3), 463-483.

Heyes, C. M., \& Foster, C. L. (2002). Motor learning by observation: Evidence from a seria reaction time task. Quarterly Journal of Experimental Psychology, 55A, 593-607.

Heyes, C. M., \& Ray, E. (2000). What is the significance of imitation in animals? Advances in the Study of Behaviour, 29, 215-245.

Heyes, C., \& Ray, E. (2004). Spatial S-R compatibility effects in an intentional imitation task. Psychonomic Bulletin \&' Review, 11, 703-708.

Hikosaka, O., Nakahara, H., Rand, M. K., Sakai, K., Lu, X., Nakamura, K., et al. (1999). Parallel neural networks for learning sequential procedures. Trends in Neurosciences, 22 464-471.

Hikosaka, O., Rand, M. K., Nakamura, K., Miyachi, S., Kitaguchi, K., Sakai, K., et al. (2002). Long-term retention of motor skill in macaque monkeys and humans. Experimental Brain Research, 147, 494-504

Holmes, M. C., \& Sholl, M. J. (2005). Allocentric coding of object-to-object relations in overlearned and novel environments. Journal of Experimental Psychology: Learning, Memory, and Cognition, 31, 1069-1087.

Howard, I. P., \& Templeton, W. B. (1966). Human spatial orientation. New York: Wiley.

Iacoboni, M., Woods, R. P., Brass, M., Bekkering, H., Mazziotta, J. C., \& Rizzolatti, G. (1999) Cortical mechanisms of human imitation. Science, 286, 2526-2528.

Ishikura, T., \& Inomata, K. (1995). Effects of angle of model-demonstration on learning of motor skill. Perceptual and Motor Skills, 80, 651-658.

Kaplan, D.M. (2013). The complex interplay between three-dimensional egocentric and allocentric spatial representation. Behavioural and Brain Sciences, 36(5), 553-554.

Keele, S. W., Jennings, P., Jones, S., Caulton, D., \& Cohen, A. (1995). On the modularity of sequence representation. Journal of Motor Behavior, 27, 17-30.

Klatzky, R. L. (1998). Allocentric and egocentric spatial representations: Definitions, distinctions, and interconnections. In C. Freksa, C. Habel, \& K. F. Wender (Eds.), Spatial cognition - An interdisciplinary approach to representation and processing of spatial knowledge (pp. 1-17). Berlin: Springer-Verlag.

Koski, L., Iacoboni, M., Dubeau, M. C., Woods, R. P., \& Mazziotta, J. C. (2003). Modulation of cortical activity during different imitative behaviours. Journal of Neurophysiology, 89, 460-471.

Koski, L., Wohlschläger, A., Bekkering, H., Woods, R. P., Dubeau, M. C., Mazziotta, J. C., et al. (2002). Modulation of motor and premotor activity during imitation of targetdirected actions. Cerebral Cortex, 12, 847-855.

Kovacs, A. J., Muehlbauer, T., \& Shea, C. H. (2009). The coding of movement sequences. Journal of Experimental Psychology: Human Perception and Performance, 35, 390-407.

Krams, M., Rushworth, M. F. S., Deiber, M. -P., Frackowiak, R. S. J., \& Passingham, R. E. (1998). The preparation, execution and suppression of copied movements in the human brain. Experimental Brain Research, 120, 386-398.

McDonough, L., Choi, S., \& Mandler, J. M. (2003). Understanding spatial relations: Flexible infants, lexical adults. Cognitive Psychology, 46, 229-259.
Meiselman, H. L., Bose, H. E., \& Nikvist, W. F. (1972). Magnitude production and magnitude estimation of taste intensity. Perception \& Psychophysics, 12, 249-252.

Meltzoff, A. N. (2002). Imitation as a mechanism of social cognition: Origins of empathy, theory of mind, and the representation of action. In U. Goshwami (Ed.), Blackwell handbook of childhood cognitive development (pp. 6-25). Oxford: Blackwell.

Mengotti, P., Corradi Dell'Acqua, C., \& Rumiati, R. I. (2012). Imitation components in the human brain: An FMRI study. NeuroImage, 59, 1622-1630.

Mou, W., Fan, Y., McNamara, T. P., \& Owen, C. (2008). Intrinsic frames of reference and egocentric viewpoints in scene recognition. Cognition, 106, 750-769.

Mou, W., McNamara, T. P., Rump, B., \& Xiao, C. (2006). Roles of egocentric and allocentric spatial representations in locomotion and reorientation. Journal of Experimental Psychology: Learning, Memory, and Cognition, 32, 1274-1290.

Mou, W., McNamara, T. P., Valiquiette, C. M., \& Rump, B. (2004). Allocentric and egocentric updating of spatial memories. Journal of Experimental Psychology: Learning, Memory, and Cognition, 30, 142-157.

Navon, D. (1987). Why do we blame the mirror for reversing left and right? Cognition, 27, 275-283.

Nishitani, N., \& Hari, R. (2000). Temporal dynamics of cortical representation for action. Proceedings of the National Academy of Sciences of the United States of America, 97. 913-918.

Osman, M., Bird, G., \& Heyes, C. (2005). Action observation supports effector-dependent learning of finger movement sequences. Experimental Brain Research, 165, 19-27.

Press, C., Ray, E., \& Heyes, C. (2009). Imitation of lateralised body movements: Doing it the hard way. Laterality, 14(5), 515-527.

Prinz, W. (2002). Experimental approaches to imitation. In A. N. Meltzoff, \& W. Prinz (Eds.), The imitative mind: Development, evolution, and brain bases (pp. 143-163). Cambridge University Press.

Proctor, R. W., \& Vu, K. -P. L. (2006). Stimulus-response compatibility principles: Data, theory and application. Boca Raton: Taylor \& Francis.

Quinn, P. C., \& Bhatt, R. S. (2005). Good continuation affects discrimination of visual pattern information in young infants. Perception \& Psychophysics, 67, 1171-1176.

Quinn, P. C., Cummins, M., Kase, J., Martin, E., \& Weisman, S. (1996). Development of categorical representations for above and below spatial relations in 3- to 7-month-old infants. Developmental Psychology, 32, 942-950.

Reynolds, G. S., \& Stevens, S. S. (1960). Binaural summation of loudness. Journal of the Acoustical Society of America, 32, 1337-1344.

Rizzolatti, G., Fadiga, L., Matelli, M., Bettinardi, V., Paulesu, E., Perani, D., et al. (1996). Localization of grasp representations in humans by PET. I. Observation versus execution. Experimental Brain Research, 111, 246-252.

Savardi, U., \& Bianchi, I. (2009). The spatial path to contrariety. In U. Savardi (Ed.), The perception and cognition of contraries (pp. 63-92). Milano, Italy: Mc-Graw Hill.

Savardi, U., Bianchi, I., \& Bertamini, M. (2010). Naive prediction of orientation and motion in mirrors. From what we see to what we expect reflections to do. Acta Psychologica, 134(1), 1-15.

Schmidt, R. A. (1975). A schema theory of discrete motor skill learning. Psychological Review, 82, 225-260.

Schofield, W. N. (1976). Do children find movements which cross the body midline difficult? Quarterly Journal of Experimental Psychology, 28, 571-582.

Sholl, M. J. (2001). The role of a self-reference system in spatial navigation. In D. Montello (Ed.), Spatial information theory: Foundations of geographical information science (pp. 217-232). Berlin, Germany: Springer-Verlag.

Sorrento, G. U., \& Henriques, D. Y. P. (2008). Reference frame conversion for repeated arm movements. Journal of Neurophysiology, 9(6), 2968-2984.

Stevens, S. S., \& Greenbaum, H. B. (1966). Regression effect in psychophysical judgment. Perception \&' Psychophysics, 1, 439-446.

Stevens, S. S., \& Guirao, M. (1962). Loudness, reciprocality, and partition scales. Journal of the Acoustical Society of America, 34, 1466-1471.

Stevens, J. C., \& Mack, J.D. (1959). Scales of apparent force. Journal of Experimental Psychology, 58, 405-413.

Takano, Y. (1998). Why does a mirror image look left-right reversed? A hypothesis of multiple processes. Psychonomic Bulletin \& Review, 5, 37-55.

Umiltà, C., \& Nicoletti, R. (1990). Spatial stimulus-response compatibility. In R. W. Proctor, \& T. G. Reeve (Eds.), Stimulus-response compatibility (pp. 89-116). Amsterdam: Elsevier Science.

Verwey, W. B. (1999). Evidence for a multi-stage model of practice in a sequential movement task. Journal of Experimental Psychology: Human Perception and Performance, 25, 1693-1708.

Waller, D., \& Greenauer, N. (2007). The role of body-based sensory information in the acquisition of enduring spatial representations. Psychological Research, 71(3), 322-332.

Wang, R. F., \& Spelke, E. S. (2000). Updating egocentric representations in human navigation. Cognition, 77, 215-250.

Wapner, S., \& Cirillo, L. (1968). Imitation of a model's hand movements: Age changes in transposition of left-right relations. Child Development, 39, 887-894.

Watanabe, R., Higuchi, T., \& Kikuchi, Y. (2013). Imitation behavior is sensitive to visual perspective of the model: an fMRI study. Experimental Brain Research, 228(2), $161-171$. 